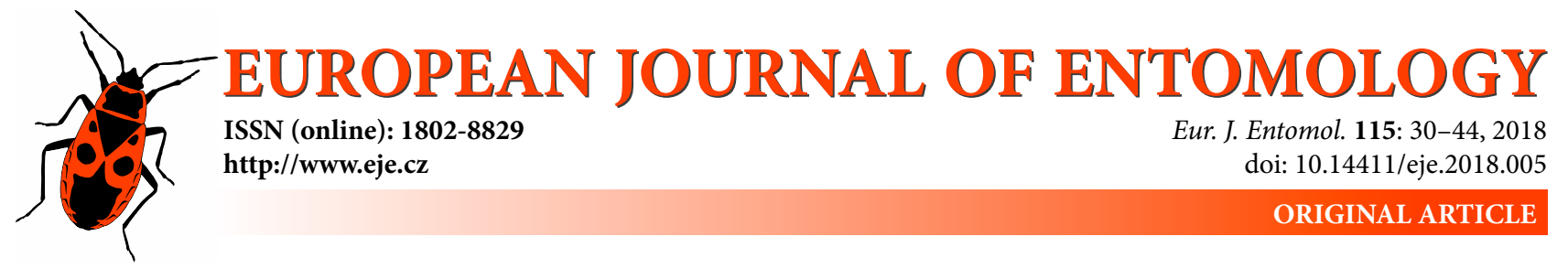

\title{
Taxonomic revision of the genus Angulaphthona (Coleoptera: Chrysomelidae: Galerucinae: Alticini)
}

\author{
MAURIZIo BIONDI and PAOLA D’ALESSANDRO* \\ University of L'Aquila, Department of Life, Health and Environmental Sciences, Division of Environmental Sciences, Via Vetoio, \\ L’Aquila 67100, Italy; e-mails: maurizio.biondi@univaq.it, paola.dalessandro@univaq.it
}

\begin{abstract}
Key words. Coleoptera, Chrysomelidae, Galerucinae, Alticini, Angulaphthona, Afrotropical region, Arabian Peninsula, Sri Lanka, taxonomy, revision, new combination, new species, new synonyms

Abstract. A revision of the genus Angulaphthona Bechyné, 1960 is provided, with a description of Angulaphthona confusa sp. $\mathrm{n}$. from the Democratic Republic of Congo, and A. rossii sp. n. from Sierra Leone. The following synonymies are proposed: Angulaphthona latipennis (Pic, 1921) = A. latipennis zambeziensis (Bechyné, 1959) syn. n. and Angulaphthona pelengensis Bechyné, $1960=A$. exalta Bechyné, 1960 syn. $\mathrm{n}$. The precedence of the name pelengensis is discussed. Angulaphthona violaceomicans (Chen, 1936) comb. n. (transferred from Aphthona) from Sri Lanka is established, and some hypotheses to explain the presence of the genus outside Africa are put forward. A key to the seven known species is supplied, with microphotographs of diagnostic characters, including male and female genitalia.
\end{abstract}

ZooBank Article LSID: A222EC5B-6130-45C8-9BB4-122AC6D6F8B2

\section{INTRODUCTION}

Alticini are a tribe of small to medium sized Coleoptera Chrysomelidae, named "flea beetles" because of the presence of a metafemoral extensor tendon that enables them to jump (Furth \& Suzuki, 1998; Nadein \& Betz, 2016). It is included within the subfamily Galerucinae, along with the closely related Galerucini (Bouchard et al., 2011). Both the adult and larval stages feed on stems, leaves or roots and rarely on flowers, of almost all the higher plant families, generally with high levels of specialization (Konstantinov \& Vandenberg, 1996). It is probably the largest and most diverse tribe of Chrysomelidae, comprising over 534 genera and about 8000 species (Nadein, 2012; Nadein \& Bezděk, 2014), occurring all over the world, and ranging from widely distributed in more than one zoogeographical region, to strictly endemic. The highest species richness occurs in the tropics in the southern hemisphere, even though our knowledge about this tribe is still incomplete for these areas (Biondi \& D'Alessandro, 2010a, 2012; Konstantinov \& Vandenberg, 1996; Nadein \& Bezděk, 2014), as demonstrated by the high number of new species still being described in recent taxonomic papers (e.g. Biondi \& D'Alessandro, 2008, 2010b; D'Alessandro et al., 2014, 2016). The whole Afrotropical region hosts about
1600 known species, included in 101 genera (Biondi, pers. data; Biondi \& D’Alessandro, 2010, 2012, 2013a, b, 2015, 2016, 2017; Biondi et al., 2017; D’Alessandro et al., 2014, 2017; Döberl, 2010), and shares a high percentage of genera with the Oriental (27\%) and Palaearctic (27\%) regions (Biondi \& D'Alessandro, 2012). The genus Angulaphthona Bechyné, 1960 was known as the unique Pan-African flea beetle genus, occurring in Mediterranean Africa (Egypt), Sub-Saharan Africa and Madagascar (Biondi \& D'Alessandro, 2012). However, previous authors included Egypt in the geographic distribution based on a misunderstanding: the type locality of Aphthona latipennis Pic (now Angulaphtona), Khartoum, originally served as an outpost for the Egyptian Army, and its history was linked to Egypt until 1956, when it became the capital of the independent Republic of Sudan.

Bechyné (1960) described the genus Angulaphthona based on some pronotal characters, and transferred to it the following species: Aphthona heteromorpha Bechyné, 1955, A. latipennis Pic, 1921 and A. zambeziensis Bechyné, 1959. In the same paper, he describes Angulaphthona exalta Bechyné, 1960 and A. pelengensis Bechyné, 1960. Later, Gruev (1981) described Angulaphthona schereri. In this contribution, we provide a revision of the genus based

\footnotetext{
* Corresponding author; e-mail: paola.dalessandro@univaq.it
} 
on new material. We describe Angulaphthona confusa sp. n. from the Democratic Republic of Congo and $A$. rossii sp. n. from Sierra Leone, and propose A. pelengensis as a synonym of $A$. exalta. We also deal with the taxonomic status and natural history of Aphthona violaceomicans Chen, 1936 from Sri Lanka, here transferred to Angulaphthona. We provide microphotographs of the main diagnostic characters of the genus and the species, including male and female genitalia, and a key to the seven known species.

\section{MATERIAL AND METHODS}

Material examined consisted of dried pinned specimens preserved in the institutions listed below. Specimens were examined, measured and dissected using a Leica M205C binocular microscope. Photomicrographs were taken using a Leica DFC500 camera and processed using Zerene Stacker version 1.04 software. Scanning electron micrographs were taken using an Hitachi TM-1000 SEM. Geographical coordinates of the localities were reported in degrees, minutes and seconds (DMS-WGS84 format); coordinates and geographic information included in square brackets were added by the authors using information from the website GoogleEarth. Chorotypes follow Biondi \& D'Alessandro (2006). The terminology for genitalia follows: Döberl (1986), Furth \& Suzuki (1994) and Suzuki (1988) for the spermatheca; D'Alessandro et al. (2016: Fig. 10E) for the median lobe of the aedeagus, and Furth \& Suzuki (1998) and Nadein \& Betz (2016) for the metafemoral extensor tendon. Ecological notes are reported in terms of African types of vegetation, primarily the divisions and formations identified and described by Sayre et al. (2013), based on the geographic coordinates of the localities where the specimens were collected.

Abbreviations. Morphology: LA - numerical sequence proportional to length of each antennomere; LAED - length of aedeagus; LAN - length of antennae; LB - total length of body (from apical margin of head to apex of elytra); LE - length of elytra; LP - medial length of pronotum; LSPC - length of spermathecal capsule; WE - maximum width of both elytra; WP - maximum width of pronotum; spec. - specimen/specimens. When possible, 10 male and 10 female specimens were measured to determine the mean, standard deviation and range for each sex.

Collections and depositories: BAQ - collection of M. Biondi, Dipartimento di Medicina clinica, Sanità pubblica, Scienze della Vita e dell'Ambiente, Università dell'Aquila, Italy; BMNH - The Natural History Museum, London, U.K.; JBCB - Jan Bezděk collection, Brno, Czech Republic; MHNB - Muséum d'Histoire Naturelle de Bâle, Bâle (= Basel), Switzerland; HNHM - Hungarian Natural History Museum, Budapest, Hungary; MNHN Museum National d'Histoire Naturelle, Paris, France; MSNG Museo Civico di Storia Naturale, Genova, Italy; MZF - Finnish Museum of Natural History, Helsinki, Finland; MZUF - Museo di Storia naturale "La Specola", Sezione di Zoologia, Università di Firenze, Italy; RMCA - Musee Royal de l'Afrique Centrale, Tervuren, Belgium; SANC - South African National Collection of Insects, Pretoria, Republic of South Africa; SDEI - Senckenberg Deutsches Entomologisches Institut, Müncheberg, Germany; ZMHB - Museum für Naturkunde der Humboldt-Universität, Berlin, Germany; ZSM - Zoologische Staatssammlung München, Germany. These internationally recognized codes follow the list of "The Insect and Spider Collections of the World Website" (Evenhuis, 2016).

\section{RESULTS}

\section{Genus Angulaphthona Bechyné, 1960}

Angulaphthona Bechyné, 1960: 74; Scherer, 1978: 265; Gruev, 1981: 55; Medvedev, 1996: 217, 261; Biondi \& D’Alessandro, 2010: 403; 2012: 25, 30.

Type species. Aphthona heteromorpha Bechyné 1955: 62 (Madagascar: Bas Mangoky), by original designation.

Distribution. Democratic Republic of Congo, Eritrea, Madagascar, Malawi, Mozambique, Nigeria, Republic of South Africa, Saudi Arabia, Sierra Leone, Somalia, Sri Lanka, Sudan, Chad, Uganda, Yemen, Zambia, Zimbabwe (Fig. 31).

Ecology. Angulaphthona heteromorpha was collected on cotton plants, Gossypium sp. (Malvaceae) (Bechyné, 1955, as Aphthona). Pollard (1957) and Gentry (1965) report Aphthona latipennis on: Abutilon pannosum, Cotton, $\mathrm{Hi}$ biscus exculentus (Malvaceae); Cajanus indicus, Dolichos lablab, Medicago sativa, Phaseolus vulgaris (Fabaceae); Calotropis procera (Apocynaceae); Aristolochia bracteolata (Aristolochiaceae); Farsetia grandiflora, Raphanus sativus (Brassicaceae); Heliotropium sp. (Boraginaceae); Sorghum vulgare (Poaceae); Eclipta alba (Asteraceae); Nicotiana tabacum (Solanaceae); Mangifera indica, Pistacia sp. (Anacardiacee); Ipomoea sp. (Convolvulaceae). Scherer (1962a) reports Aphthona latipennis from "galerie forestière".

Remarks. Angulaphthona is identifiable by several well-defined characters that are easily recognizable in all species, which make it easy to distinguish it from similar genera, specifically Aphthona Chevrolat, 1836, which is considered to be the closest (Bechyné, 1960). The main diagnostic characters (basis for the specific epithet) regards the basal margin of pronotum, laterally slightly expanded and raised, with posterior angles bearing a tubercle with a setigerous pore (Figs 8-15) (without any lateral expansion and tubercle in Aphthona). Pronotum is basically sub-trapezoidal, straight or slightly rounded and distinctly bordered laterally, with anterior angles thickened and generally sharpened (Figs 8-15) (sub-rectangular, more distinctly rounded and finely bordered laterally, with anterior angles neither thickened nor sharpened in Aphthona). Other useful diagnostic characters shared by all species are: frontal tubercles V-shaped (Fig. 16) (generally sub-triangular or roundish in Aphthona); frontal carina clearly raised, apically rounded, expanded above the clypeus (Fig. 16); elytra distinctly bordered laterally, with confused and generally clearly impressed punctation (Figs 8-15) (narrowly bordered laterally, generally with slightly or moderately impressed punctation in Aphthona); spermatheca with coiled ductus (Figs 25-30) (generally uncoiled in Aphthona, very rarely coiled). Dorsal integuments are always blue or blackish, with distinct metallic blue reflections (Figs 1-7). No special diagnostic characters are found on legs. The metafemoral extensor tendon of Angulaphthona (Fig. 17) can be attributed to the Psylliodes Morpho-Group (Furth \& Suzuki, 1998) by having: the basal angle of the ventral 

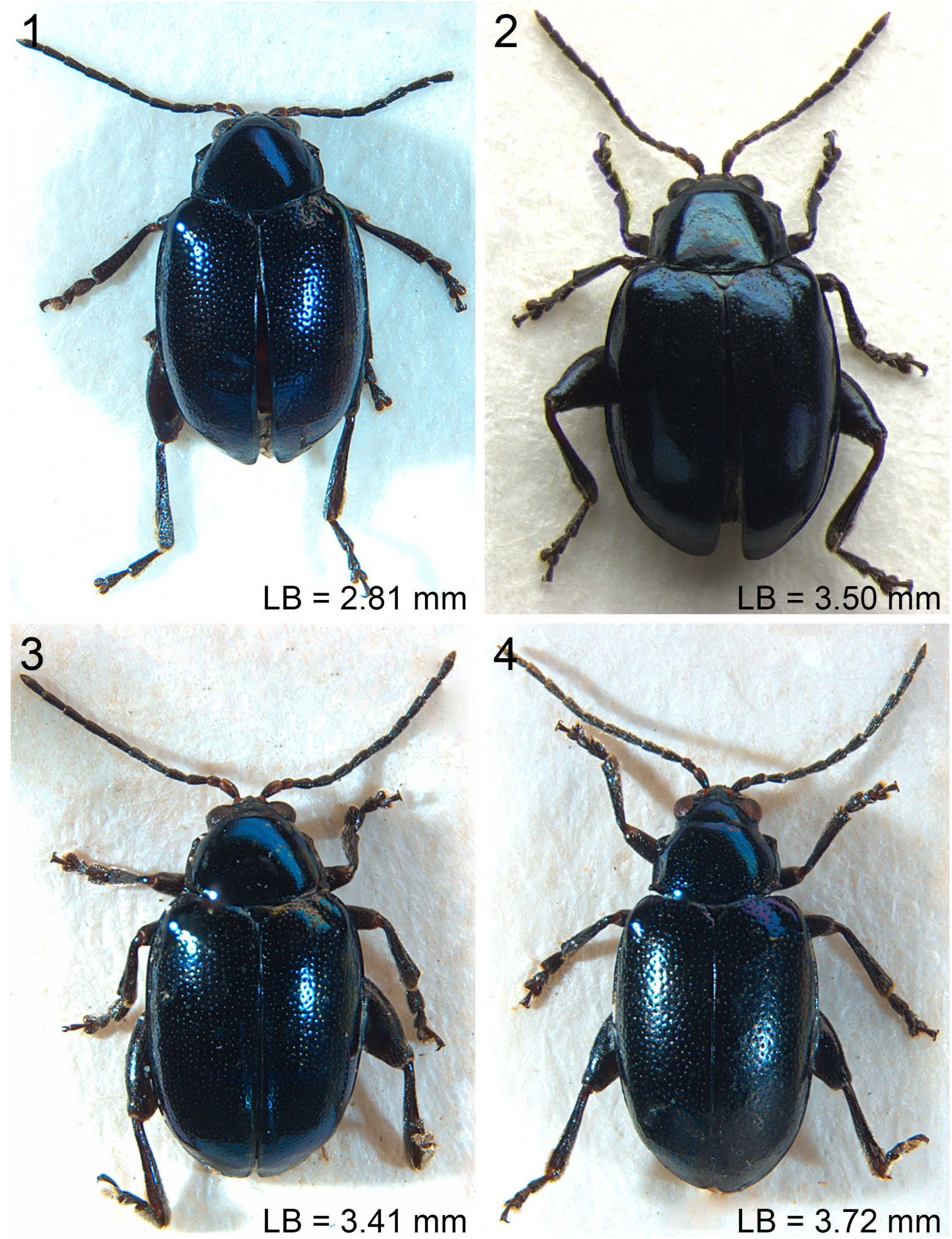

Figs 1-4. Habitus. 1 - Angulaphthona confusa sp. n., holotype, male; $2-A$. heteromorpha, female, Madagascar, Berenty Reserve; $3-A$. latipennis, male, Mozambique, Monapo; 4 - A. pelengensis, male, Uganda, Semliki Forest. LB - body length.

lobe very narrow (acute) and pointed; a very distinct recurved flange; a flat tendon basal edge which is at about a right angle to the central axis of the dorsal lobe; however, the extended arm is not very depressed. The metafemoral extensor tendon of Aphthona (Furth, 1980: Fig. 6) belongs to the Chaetocnema Morpho-Group that is similar to the Psylliodes Morpho-Group, but with dorsal edge of ventral lobe strongly angled down and without the recurved flange
(Furth, 1980; Furth \& Suzuki, 1998). Pronotum bears both diagnostic characters for the genus, and features for distinguishing the species. However, the main diagnostic characters at the species level are associated with the genitalia. The median lobe of the aedeagus of each species is easily recognizable (Figs 18-24). Spermatheca can be attributed to two distinct groups, depending on whether the apical and basal part are well separated or not (Figs 25-30); the 

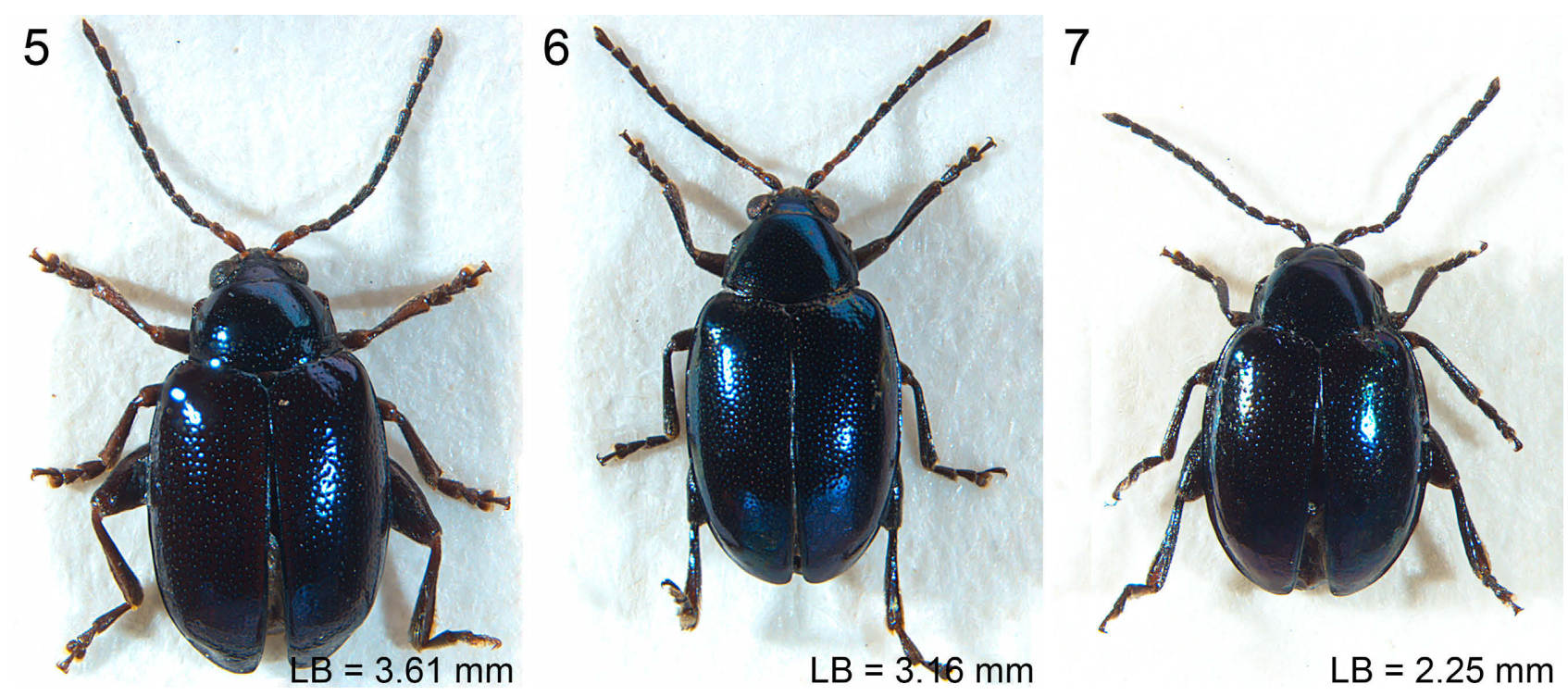

Figs 5-7. Habitus. 5 - Angulaphthona rossii sp. n., holotype, male; 6 - A. schereri, paratype, male, Yemen, Wadi Zabid; 7 - A. violaceomicans, male, Sri Lanka. LB - body length.

only two species with separated apical and basal parts $(A$. schereri and $A$. violaceomicans) also have a short appendix on the distal part. The sexual dimorphism concerns the size of first pro- and mesotarsomere, more or less enlarged in males of most species, and the sculpture on the last visible abdominal ventrite, which has a central lobe with an evident median carina in males of five out of seven species. Interestingly, the only two species that have a simple last ventrite, $A$. schereri and $A$. violaceomicans, also have similar spermathecae.

\section{ANNOTATED CATALOGUE OF THE SPECIES}

\section{Angulaphthona confusa sp. $\mathbf{n}$.}

(Figs 1, 8, 18, 28, 31)

ZooBank taxon LSID:

B714B67F-132F-4D6F-806F-9D51DED7B77C

Diagnosis. Angulaphthona confusa sp. n. can be identified by the following pronotal characters (Fig. 8): posterior and lateral margins rounded (especially in male); anterior angles clearly sharpened; punctation dense, uniform and small, clearly smaller than on elytra. Median lobe of aedeagus (Fig. 18) is very peculiar in having an elongate, apically rounded apical extension. Spermatheca is damaged (it lacks the ductus) (Fig. 28), but its capsule is very similar to those of $A$. heteromorpha, A. latipennis and $A$. pelengensis, as the apical and basal parts are not separated (Figs 19-21).

Description of the holotype $(\delta)$. Body sub-elliptical, moderately convex (Fig. 1$) ; \mathrm{LB}=2.81 \mathrm{~mm}$; maximum pronotal width in the middle ( $\mathrm{WP}=1.05 \mathrm{~mm})$; maximum elytral width in the middle $(\mathrm{WE}=1.63 \mathrm{~mm})$. Dorsum dark blue, with metallic reflection. Head with smooth surface, very finely micropunctate (Fig. 8); frontal carina moderately raised, quite thin, rounded apically, continuous with post-clypeal area; frontal tubercles clearly elongate, Vshaped, weakly raised, clearly delimited only posteriorly; frontal grooves distinctly impressed, from frontal tubercles to upper ocular margin. Antennae distinctly longer than half body length (LAN = $1.94 \mathrm{~mm}$; LAN/LB = 0.69), dark brown, mostly homogenous in colour; LA: 100:71:94:106: 106:100:106:118:106:106:147. Pronotum (Fig. 8) subtrapezoidal, moderately transverse $(\mathrm{LP}=0.75 \mathrm{~mm}$; WP/LP $=1.40$ ), weakly rounded laterally, with clearly expanded lateral margins; basal margin distinctly rounded; anterior angles clearly prominent and sharpened; posterior angles with flat margins and distinctly prominent setigerous pore; pronotal surface weakly micropunctate, almost smooth, with dense, small and uniform punctation. Metathoracic wings macropterous. Elytra moderately elongate (LE = $2.27 \mathrm{~mm} ; \mathrm{WE} / \mathrm{LE}=0.72$ ), laterally distinctly rounded (Fig. 1); punctation confused, more strongly impressed than on pronotum (Fig. 8). Legs brownish; first pro- and mesotarsomeres moderately dilated. Venter blackish; last visible abdominal ventrite with central lobe bearing a distinct median carina. Aedeagus (LAED = $1.19 \mathrm{~mm}$; LE/LAED $=1.91$ ) (Fig. 18) in ventral view slightly enlarged in apical part, with strongly elongate, apically rounded apex; ventral sulcus short, sub-elliptical; in lateral view median lobe slightly curved, with dorsally bent apex; sulcus of dorsal ligula short, placed on sub-apical part.

Variation. Female $(\mathrm{n}=1): \mathrm{LE}=2.41 \mathrm{~mm} ; \mathrm{WE}=1.78 \mathrm{~mm}$; $\mathrm{LP}=0.69 \mathrm{~mm} ; \mathrm{WP}=1.06 \mathrm{~mm} ; \mathrm{LAN}=2.03 \mathrm{~mm} ; \mathrm{LSPC}=0.38$ $\mathrm{mm} ; \mathrm{LB}=2.94 \mathrm{~mm} ; \mathrm{LE} / \mathrm{LP}=3.50 ; \mathrm{WE} / \mathrm{WP}=1.68 ; \mathrm{WP} / \mathrm{LP}=$ 1.55; WE/LE $=0.74 ; \mathrm{LAN} / \mathrm{LB}=0.69 ; \mathrm{LE} / \mathrm{LSPC}=6.42$. Paratype very similar in size, shape, sculpture and colour to the holotype. Female with posterior margin of pronotum slightly less rounded, and first anterior and median tarsomeres not dilated. Spermatheca (Fig. 28) with apical and basal part not distinct; ductus damaged.

Type material. Holotype $\delta$ : Congo belge [Democratic Republic of Congo]: ex P.N.U. Kiamakoto entre Masombwe-Mukana, r.dr. Lukima, af. dr. Gr. Kafwe, $1070 \mathrm{~m}\left[9^{\circ} 09^{\prime} 05.29^{\prime \prime} \mathrm{S}\right.$, $27^{\circ} 10^{\prime} 37.21^{\prime \prime E}$ ], 20.ix.1948, Mis. G.F. de Witte. 1841a", paratype of Angulaphthona pelengensis. Paratype: 1 , , same data as the holotype, paratype of Angulaphthona pelengensis (RMCA). 

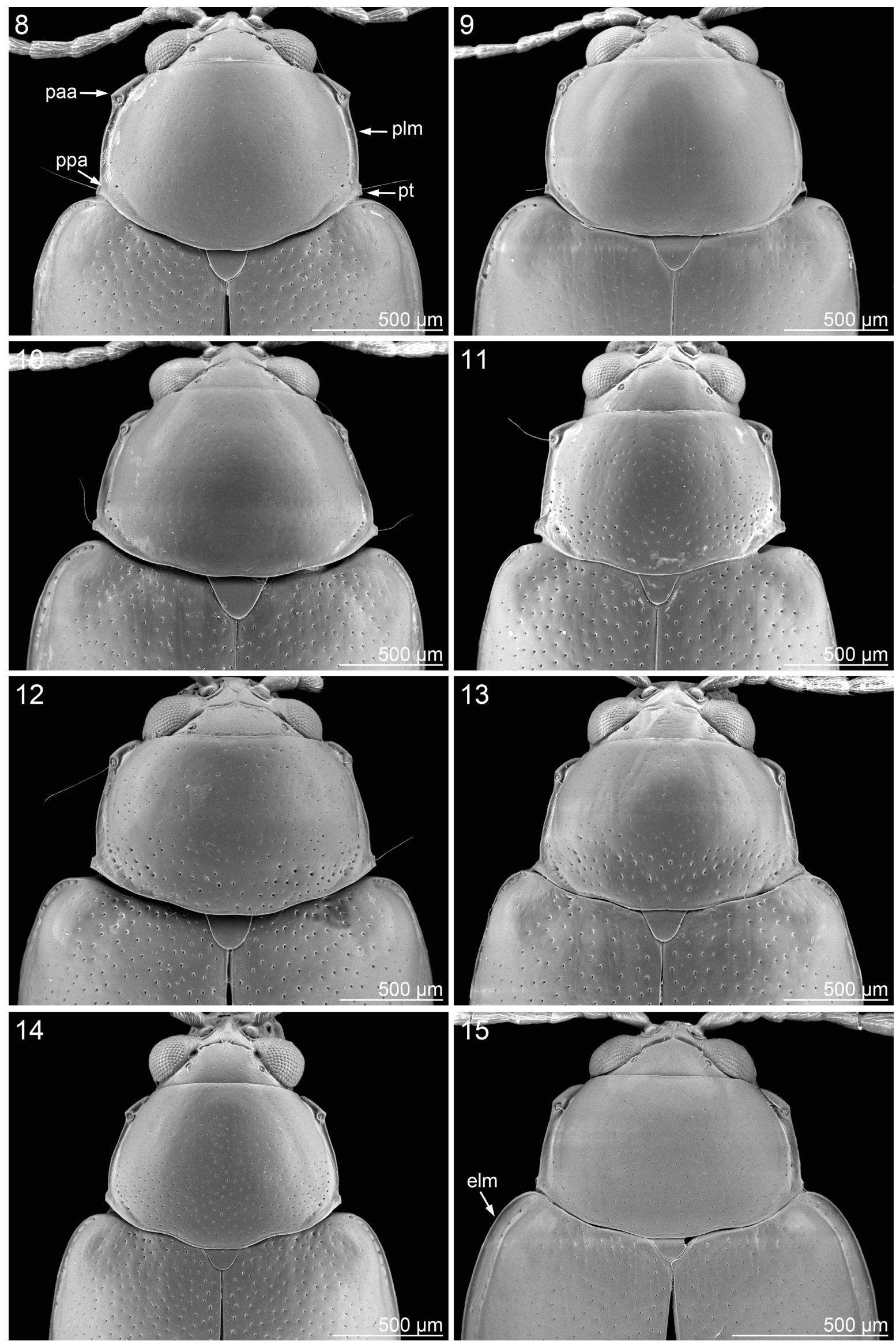

Figs 8-15. Head, pronotum and elytral base. 8 - Angulaphthona confusa sp. n.; 9 - A. heteromorpha; $10-A$. latipennis; $11-A$. pelengensis; 12 - ditto (holotype of $A$. exalta); 13 - A. rossii sp. n.; $14-A$. schereri; $15-A$. violaceomicans. elm - lateral margin of elytra; paa - anterior angle of pronotum; plm - lateral margin of pronotum; ppa - posterior angle of pronotum; pt - pronotal tubercle. 

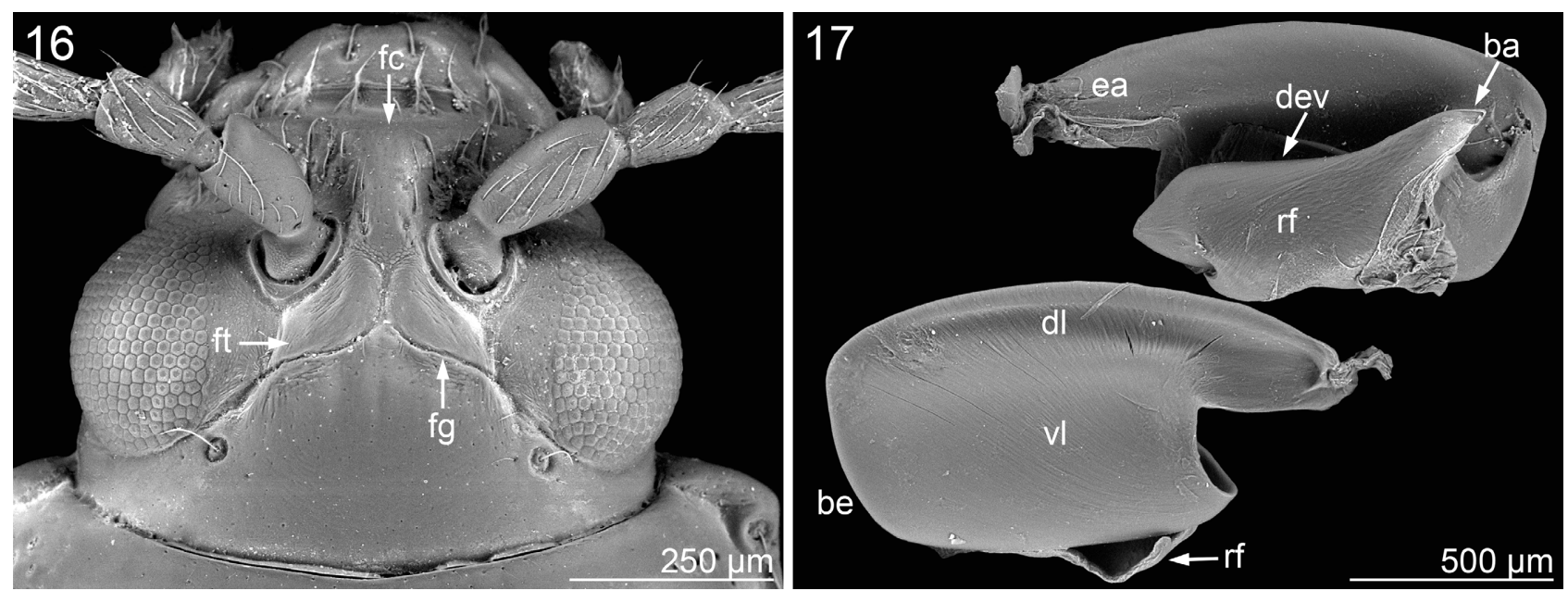

Figs 16, 17. Characters of Angulaphthona. 16 - frons and vertex of $A$. pelengensis; 17 - right metafemoral extensor tendon of $A$. schereri, anterior (top) and posterior (bottom) view. ba - basal angle of the ventral lobe; be - tendon basal edge; dev - dorsal edge of ventral lobe; $\mathrm{dl}$ - dorsal lobe; ea - extended arm; fc - frontal carina; $\mathrm{fg}$ - frontal groove; $\mathrm{ft}$ - frontal tubercle; $\mathrm{rc}$ - recurved flange; $\mathrm{vl}$ - ventral lobe.

Type locality. Kiamakoto (Democratic Republic of Congo).

Etymology. The specific epithet is a Latin adjective meaning "confused", "mixed up", and referring to its previous attribution to the species $A$. pelengensis.

Distribution. Democratic Republic of Congo (Fig. 31). Central Afrotropical (CAT) chorotype.

Ecological notes. Not available. The collecting site falls within the area of Miombo \& Associated Broadleaf Savanna vegetation (belonging to the Tropical Lowland Grassland, Savanna \& Shrubland formation) (Sayre et al., 2013).

\section{Angulaphthona heteromorpha (Bechyné, 1955)}

(Figs 2, 9, 19, 26, 31)

Aphthona heteromorpha Bechyné, 1955: 62; Bechyné, 1959: 230. Angulaphthona heteromorpha: Bechyné, 1960: 74; Gruev, 1981: 56.

Type material examined. Holotype $\delta$ : Madagascar, E.DC.M.10, ex cotonnier feuille, Bas Mangoky [21 ${ }^{\circ} 35^{\prime} 35.22^{\prime \prime S}$, $\left.43^{\circ} 43^{\prime} 25.79^{\prime \prime} \mathrm{E}\right](\mathrm{MNHN})$. Allotype: $1{ }^{\circ}$, same data as holotype (MNHN).

Type locality. Bas Mangoky (Madagascar).

Additional material examined. Madagascar: Boeni, Basse Betsiboka, Marovoay [1606 $26.48^{\prime \prime S}, 46^{\circ} 38^{\prime} 47.03^{\prime \prime}$ ] ], 5.i.1900, Dr. J. De Corse 1901, 1 spec. (MNHN); Berenty Reserve nr. Amboasary $\left[24^{\circ} 59^{\prime} 52.63^{\prime \prime} \mathrm{S}, 46^{\circ} 19^{\prime} 52.64^{\prime \prime E}\right]$, 19.x.1970, sweeping river bank, 1 spec. (BMNH); Maj., Ambato-Boeni [ $16^{\circ} 28^{\prime} 15.20^{\prime \prime}$, 464' 59.13"E], 23.vi.1958, F. Keiser leg., 1 spec. (MHNB);

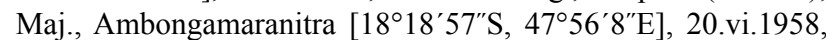
F. Keiser leg., 1 spec. (MHNB).

Remarks. Angulaphthona heteromorpha is distinguishable by both the pronotal and elytral punctation, clearly smaller and shallower compared to other species (Fig. 9); lateral margins of pronotum generally slightly curved and weakly expanded (but more expanded anteriorly in some specimens). Median lobe of aedeagus (Fig. 19) in ventral view with two lateral, pointed expansions apically, and a large, rounded, flattened median tooth; in lateral view median lobe mostly thick and clearly curved; dorsal surface with an elongate, deep, V-shaped sulcus, bearing two lateral expansions apically. Spermatheca (Fig. 26) with api- cal and basal part not definitely separated; ductus inserted apically, closely coiled over most of its length. In some specimens spermatheca with wider basal part, and slightly thinner ductus, more clearly and unevenly coiled.

Biometry. Male $(\mathrm{n}=1$, holotype): $\mathrm{LE}=2.97 \mathrm{~mm} ; \mathrm{WE}=2.05$ $\mathrm{mm} ; \mathrm{LP}=0.91 \mathrm{~mm} ; \mathrm{WP}=1.33 \mathrm{~mm} ; \mathrm{LAN}=2.34 \mathrm{~mm} ; \mathrm{LAED}=$ $1.19 \mathrm{~mm} ; \mathrm{LB}=3.66 \mathrm{~mm} ; \mathrm{LE} / \mathrm{LP}=3.28 ; \mathrm{WE} / \mathrm{WP}=1.54 ; \mathrm{WP} /$ $\mathrm{LP}=1.47 ; \mathrm{WE} / \mathrm{LE}=0.69 ; \mathrm{LAN} / \mathrm{LB}=0.64 ; \mathrm{LE} / \mathrm{LAED}=2.50$. Female ( $\mathrm{n}=5$; mean and standard deviation; range): $\mathrm{LE}=2.85 \pm$ $0.13 \mathrm{~mm}(2.69 \leq \mathrm{LE} \leq 3.00 \mathrm{~mm}) ; \mathrm{WE}=2.11 \pm 0.11 \mathrm{~mm}(2.02 \leq$ $\mathrm{WE} \leq 2.28 \mathrm{~mm}) ; \mathrm{LP}=0.85 \pm 0.06 \mathrm{~mm}(0.78 \leq \mathrm{LP} \leq 0.92 \mathrm{~mm})$; $\mathrm{WP}=1.28 \pm 0.06 \mathrm{~mm}(1.22 \leq \mathrm{WP} \leq 1.38 \mathrm{~mm}) ; \mathrm{LAN}=2.26 \pm$ $0.18 \mathrm{~mm}(2.00 \leq \mathrm{LAN} \leq 2.44 \mathrm{~mm}) ; \mathrm{LSPC}=0.40 \pm 0.02 \mathrm{~mm}(0.38$ $\leq \mathrm{LSPC} \leq 0.42 \mathrm{~mm}) ; \mathrm{LB}=3.53 \pm 0.15 \mathrm{~mm}(3.41 \leq \mathrm{LB} \leq 3.78$ $\mathrm{mm}) ; \mathrm{LE} / \mathrm{LP}=3.34 \pm 0.12(3.18 \leq \mathrm{LE} / \mathrm{LP} \leq 3.46) ; \mathrm{WE} / \mathrm{WP}=1.65$ $\pm 0.05(1.59 \leq \mathrm{WE} / \mathrm{WP} \leq 1.73) ; \mathrm{WP} / \mathrm{LP}=1.50 \pm 0.06(1.43 \leq \mathrm{WP} /$ $\mathrm{LP} \leq 1.58) ; \mathrm{WE} / \mathrm{LE}=0.74 \pm 0.01(0.73 \leq \mathrm{WE} / \mathrm{LE} \leq 0.76) ; \mathrm{LAN} /$ $\mathrm{LB}=0.64 \pm 0.04(0.59 \leq \mathrm{LAN} / \mathrm{LB} \leq 0.69) ; \mathrm{LE} / \mathrm{LSPC}=7.12 \pm$ $0.31(6.59 \leq \mathrm{LE} / \mathrm{LSPC} \leq 7.38)$.

Distribution. Madagascar (Fig. 31). Malagasy (MAL) chorotype.

Ecological notes. Collected on cotton. No other ecological notes are available. The collecting sites are located in very different types of vegetation (Sayre et al., 2013).

\section{Angulaphthona latipennis (Pic, 1921)}

(Figs 3, 10, 17, 20, 27, 31)

Aphthona latipennis Pic, 1921: 15; Heikertinger \& Csiki, 1940; Heikertinger, 1944: 88; Bryant, 1957: 359; Pollard, 1957; 76; Bechyné, 1959: 229-230; Scherer, 1959: 189; 1961: 173; 1962a: 9; Alfieri, 1976: 234.

Angulaphthona latipennis: Bechyné, 1960: 74; Scherer, 1978: 265; Gruev, 1981: 56; Medvedev, 1996: 261.

= Aphthona zambeziensis Bechyné, 1959, syn. $\mathrm{n}$.

Aphthona zambeziensis Bechyné, 1959: 230; Scherer, 1962b: 16. Angulaphthona zambeziensis: Bechyné, 1960: 74.

Angulaphthona latipennis zambeziensis: Gruev, 1981: 56.

Type material examined. Paratype of Angulaphthona zambeziensis: 1 으. Mozambique: Zambesi, Boroma $\left[17^{\circ} 27^{\prime} 00^{\prime \prime} \mathrm{S}\right.$, $\left.35^{\circ} 40^{\prime} 00^{\prime \prime} \mathrm{E}\right], 1955$ (ZSM). 

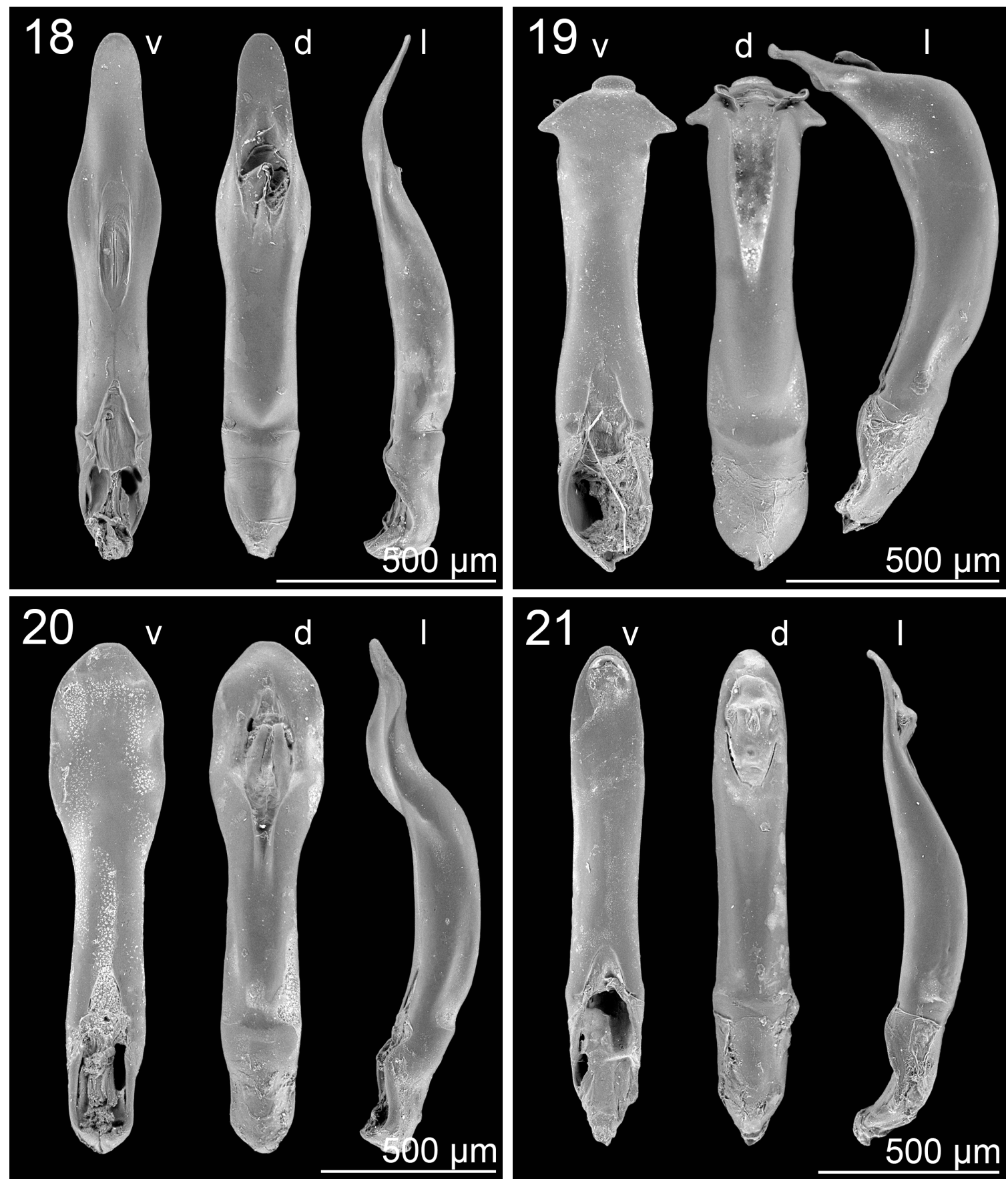

Figs 18-21. Median lobe of aedeagus in ventral (v), dorsal (d) and lateral (I) view. 18 - Angulaphthona confusa sp. n., holotype; $19-A$. heteromorpha, holotype; 20 - A. latipennis, Yemen, Wadi Zabid; 21 - A. pelengensis, holotype.

Type locality. Khartoum (Sudan).

Additional material examined. Democratic Republic of Congo: P.N.U. Kiamokoto-Kiwakishi (1.070 m) [909'34.53"S, $\left.27^{\circ} 11^{\prime} 23.40^{\prime \prime E}\right]$, 4.-16.x.1948, Mis. G.F. de Witte. 1886a, 1 spec., paratype of Angulaphthona exalta (RMCA); Eritrea:

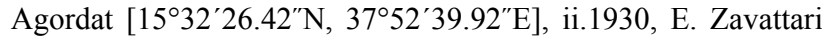
leg., 7 spec. (MSNG); Malawi: Nyasaland [= Malawi], Mlanje [1601'30.84"S, 35³0'29.93"E], 1.xii.1913, S.A. Neave leg., 1914-156, 1 spec. (BMNH); Mozambique: Port. E. Africa, Mo-

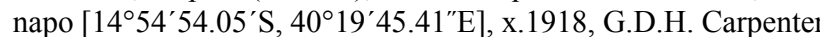
leg., 1919-36, 1 spec. (BMNH); Caia, Zambesi [1749'03.67"S, 3520'28.10"E], H. Swale leg., 1913-117, 2 spec. (BMNH); ditto, 12.xi.1910, 1 spec. (BMNH); ditto, 5.viii.1911, 1 spec. (BMNH);

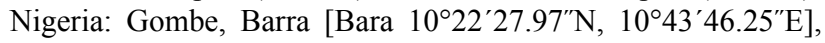

i.1929, W. Woud leg. (BMNH); Republic of South Africa: Durban [29 $\left.52^{\prime} 06.50^{\prime \prime} \mathrm{S}, 31^{\circ} 00^{\prime} 42.87^{\prime \prime} \mathrm{E}\right]$, F. Muir leg., 1905313, 2 spec. (BMNH); Saudi Arabia: Jizan prov., Wadi Atoud, $17^{\circ} 48^{\prime} \mathrm{N}, 42^{\circ} 22^{\prime} \mathrm{E}, 245$ m, 8.ii.2016, J. Bezděk \& D. Král leg., 2 spec. (JBCB); Somalia: Balad [ $\left.2^{\circ} 21^{\prime} 30.22^{\prime \prime} \mathrm{N}, 45^{\circ} 23^{\prime} 10.64^{\prime \prime} \mathrm{E}\right]$, 28.ix.1986 (S.B.S.), L. Bartolozzi leg., 2 spec. (MZUF); Mogadi-

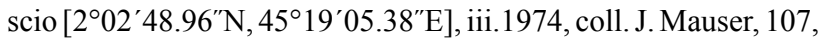
1 spec. (BAQ); Sudan: Singa [12 $\left.{ }^{\circ} 45^{\prime} 55.08^{\prime \prime} \mathrm{S}, 33^{\circ} 37^{\prime} 03.28^{\prime \prime} \mathrm{E}\right]$, on cotton, 9.xii.1926, W. Ruttledge leg., 1 spec. (BMNH); Yemen: Wadi Zabid $\left[14^{\circ} 07^{\prime} 53.26^{\prime \prime} \mathrm{N}, 43^{\circ} 31^{\prime} 46.54^{\prime \prime} \mathrm{E}\right], 1970$, A. SzalayMarzsó leg., 6 spec. (HNHM); Wadi Jaira, tributary of Wadi Siham, ca. 3,000 ft [14²1 $\left.57^{\prime \prime} \mathrm{N}, 43^{\circ} 33^{\prime} 40^{\prime \prime} \mathrm{E}\right], 10$. iii.1938, H. Scott \& E.B. Britton leg., 4 spec. (BMNH); Usaifira, 1 mile $\mathrm{N}$ of Ta'izz, ca. $4,500 \mathrm{ft}\left[13^{\circ} 37^{\prime} 05.83^{\prime \prime} \mathrm{N}, 44^{\circ} 02^{\prime} 08.46^{\prime \prime} \mathrm{E}\right]$, in cultivated 

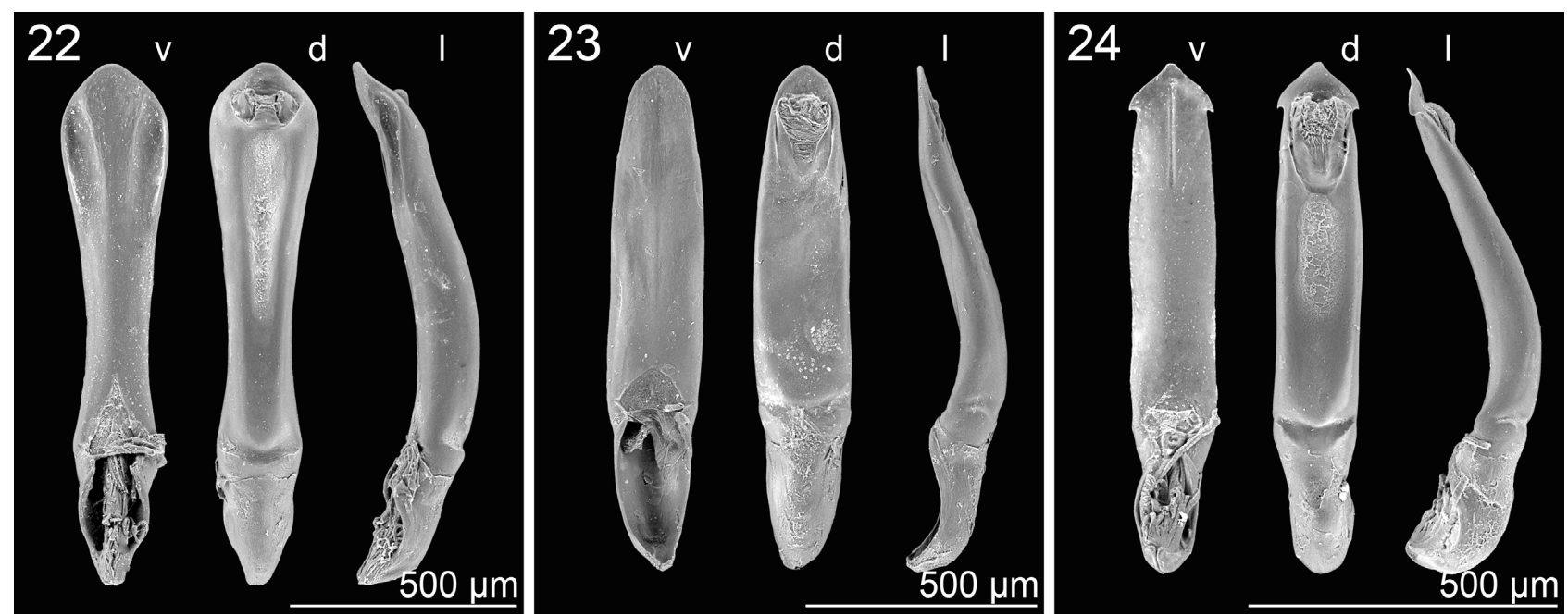

Figs 22-24. Median lobe of aedeagus in ventral (v), dorsal (d) and lateral (I) view. 22 - Angulaphthona.rossii sp. n., holotype; $23-A$. schereri, holotype; 24 - A. violaceomicans, Sri Lanka.

fields, 13.xii.1937, H. Scott \& E.B. Britton leg., 19 spec. (BMNH);

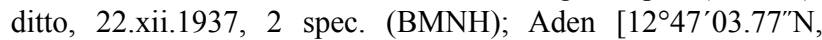
$\left.45^{\circ} 01^{\prime} 01.87^{\prime \prime} \mathrm{E}\right]$, Sharp Coll. 1905-313, 1 spec. (BMNH); Zimbabwe: N.W. Rhodesia, Lukanga R. Dist. Lukanga, $27^{\circ}-28^{\circ} \mathrm{E}$

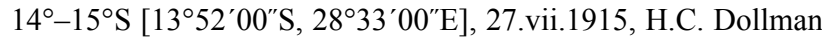
leg., 1 spec. (BMNH); Mwengwa, $27^{\circ} 40^{\prime} \mathrm{E}, 13^{\circ} \mathrm{S}\left[15^{\circ} 17^{\prime} 00^{\prime \prime} \mathrm{S}\right.$, 25 59'00"E], 24.vii.1913, H.C. Dollman leg., 1 spec. (BMNH); Chimutsi Dam, Zambesi Valley, $15^{\circ} 12^{\prime} \mathrm{S}, 2^{\circ} 13^{\prime} \mathrm{E}$, viii.-ix.1988, J. Weyrich leg., 2 spec. (SANC).

Remarks. Angulaphthona latipennis is generally distinguishable by its distinctly trapezoidal pronotum, with dense, homogenous, small punctation, clearly smaller than on elytra (Fig. 10). Median lobe of aedeagus (Fig. 21) in ventral view clearly wider in apical $2 / 5 \mathrm{~s}$, with concave surface; apex sub-truncated; in lateral view curved in the basal $2 / 3$ s, then slightly sinuate; sulcus of dorsal ligula with Vshaped base at about half length of aedeagus. Spermatheca (Fig. 27) slender, with basal and distal part not definitely separated; ductus inserted apically, widely and irregularly coiled in proximal part. Based on their identical genitalia and very similar external morphology, and taking into consideration the absence of any geographic separation, we propose the following synonymy: Angulaphthona latipennis $(\mathrm{Pic}, 1921)=$ A. latipennis zambeziensis (Bechyné, 1959) syn. n.

Biometry. Male ( $\mathrm{n}=10$; mean and standard deviation; range): $\mathrm{LE}=2.85 \pm 0.15 \mathrm{~mm}(2.56 \leq \mathrm{LE} \leq 3.06 \mathrm{~mm}) ; \mathrm{WE}=2.06 \pm 0.09$ $\mathrm{mm}(1.88 \leq \mathrm{WE} \leq 2.22 \mathrm{~mm}) ; \mathrm{LP}=0.88 \pm 0.05 \mathrm{~mm}(0.78 \leq \mathrm{LP} \leq$ $0.97 \mathrm{~mm}) ; \mathrm{WP}=1.28 \pm 0.06 \mathrm{~mm}(1.19 \leq \mathrm{WP} \leq 1.34 \mathrm{~mm}) ; \mathrm{LAN}$ $=2.38 \pm 0.13 \mathrm{~mm}(2.13 \leq \mathrm{LAN} \leq 2.59 \mathrm{~mm}) ; \mathrm{LAED}=1.52 \pm 0.07$ $\mathrm{mm}(1.44 \leq \mathrm{LAED} \leq 1.66 \mathrm{~mm}) ; \mathrm{LB}=3.55 \pm 0.16 \mathrm{~mm}(3.34 \leq \mathrm{LB}$ $\leq 3.72 \mathrm{~mm}) ; \mathrm{LE} / \mathrm{LP}=3.23 \pm 0.11(3.07 \leq \mathrm{LE} / \mathrm{LP} \leq 3.37) ; \mathrm{WE} / \mathrm{WP}$ $=1.61 \pm 0.05(1.53 \leq \mathrm{WE} / \mathrm{WP} \leq 1.66) ; \mathrm{WP} / \mathrm{LP}=1.45 \pm 0.05(1.39$ $\leq \mathrm{WP} / \mathrm{LP} \leq 1.54) ; \mathrm{WE} / \mathrm{LE}=0.72 \pm 0.02(0.70 \leq \mathrm{WE} / \mathrm{LE} \leq 0.75)$; $\mathrm{LAN} / \mathrm{LB}=0.67 \pm 0.03(0.61 \leq \mathrm{LAN} / \mathrm{LB} \leq 0.71) ; \mathrm{LE} / \mathrm{LAED}=$ $1.87 \pm 0.08(1.73 \leq \mathrm{LE} / \mathrm{LAED} \leq 1.98)$. Female $(\mathrm{n}=10$; mean and standard deviation; range): $\mathrm{LE}=2.93 \pm 0.17 \mathrm{~mm}(2.63 \leq \mathrm{LE}$ $\leq 3.19 \mathrm{~mm}) ; \mathrm{WE}=2.11 \pm 0.11 \mathrm{~mm}(1.91 \leq \mathrm{WE} \leq 2.27 \mathrm{~mm}) ; \mathrm{LP}$ $=0.85 \pm 0.06 \mathrm{~mm}(0.81 \leq \mathrm{LP} \leq 0.97 \mathrm{~mm}) ; \mathrm{WP}=1.30 \pm 0.09 \mathrm{~mm}$ $(1.16 \leq \mathrm{WP} \leq 1.41 \mathrm{~mm}) ; \mathrm{LAN}=2.28 \pm 0.09 \mathrm{~mm}(2.16 \leq \mathrm{LAN} \leq$ $2.39 \mathrm{~mm}) ; \mathrm{LSPC}=0.40 \pm 0.02 \mathrm{~mm}(0.38 \leq \mathrm{LSPC} \leq 0.45 \mathrm{~mm})$;
$\mathrm{LB}=3.58 \pm 0.17 \mathrm{~mm}(3.28 \leq \mathrm{LB} \leq 3.84 \mathrm{~mm}) ; \mathrm{LE} / \mathrm{LP}=3.44 \pm$ $0.14(3.23 \leq \mathrm{LE} / \mathrm{LP} \leq 3.65) ; \mathrm{WE} / \mathrm{WP}=1.63 \pm 0.06(1.51 \leq \mathrm{WE} /$ $\mathrm{WP} \leq 1.74) ; \mathrm{WP} / \mathrm{LP}=1.52 \pm 0.06(1.42 \leq \mathrm{WP} / \mathrm{LP} \leq 1.59) ; \mathrm{WE} /$ $\mathrm{LE}=0.72 \pm 0.02(0.68 \leq \mathrm{WE} / \mathrm{LE} \leq 0.74) ; \mathrm{LAN} / \mathrm{LB}=0.64 \pm 0.03$ $(0.61 \leq \mathrm{LAN} / \mathrm{LB} \leq 0.70) ; \mathrm{LE} / \mathrm{LSPC}=7.28 \pm 0.54(6.52 \leq \mathrm{LE} /$ LSPC $\leq 8.50$ ).

Distribution. Democratic Republic of Congo, Eritrea, Malawi, Mozambique, Nigeria, Republic of South Africa, Saudi Arabia (?), Somalia, Sudan, Chad (?), Yemen, Zimbabwe (Fig. 31). Eastern Afrotropical (EAF) chorotype.

Ecological notes. Aphthona latipennis is reported from many different plants by Pollard (1957) and Gentry (1965), and from "galerie forestière" by Scherer (1962a) (see "Ecology" in the characteristic of the genus above). Considering the checked localities, the species was collected in cultivated fields and on cotton, mainly in the Miombo \& Associated Broadleaf Savanna and Eastern \& Southern African Dry Savanna \& Woodland vegetation divisions (belonging to the Tropical Lowland Grassland, Savanna \& Shrubland formation) (Sayre et al., 2013).

\section{Angulaphthona pelengensis Bechyné, 1960}

(Figs 4, 11, 12, 16, 21, 25, 31)

Angulaphthona pelengensis Bechyné, 1960: 74; Gruev, 1981: 57. = Angulaphthona exalta Bechyné, 1960, syn. n.

Angulaphthona exalta Bechyné, 1960: 74; Gruev, 1981: 56.

Type material examined. Holotype $\odot$ of Angulaphthona exalta: Congo belge [Democratic Republic of Congo]: P.N.U. Kabwe s/Muye af. dr. Lufira (1.320 m) [8 $8^{\circ} 9^{\prime} 47.64^{\prime \prime S}, 27^{\circ} 00^{\prime} 13.68^{\prime \prime} \mathrm{E}$, 20.-25.v.1948, Mis. G.F. de Witte. 1636a (RMCA). Holotype $\delta$ of Angulaphthona pelengensis: Congo belge [Democratic Republic of Congo]: P.N.U. Gorges de la Pelenge $(1.150 \mathrm{~m})$ [2²6'31.62"S, 22 $23^{\prime} 40.09^{\prime \prime}$ E], 10.-14.vi.1947, Mis. G.F. de Witte. 474a (RMCA).

Type locality. Gorges de la Pelenge (Democratic Republic of Congo).

Additional material examined. Democratic Republic of Congo: Basoko [1 $\left.{ }^{\circ} 14^{\prime} 26.98^{\prime \prime} \mathrm{N}, 2^{\circ} 36^{\prime} 41.53^{\prime \prime} E\right]$, x.1948, P.L.G. Benoit leg., 1 spec. (RMCA). Uganda: Semliki Forest, $0^{\circ} 48^{\prime} \mathrm{N}$, $30^{\circ} 08^{\prime} \mathrm{E}$, 5.-12.ii.1997, Th. Wagner leg., $1 \mathrm{spec}$. (BAQ). 


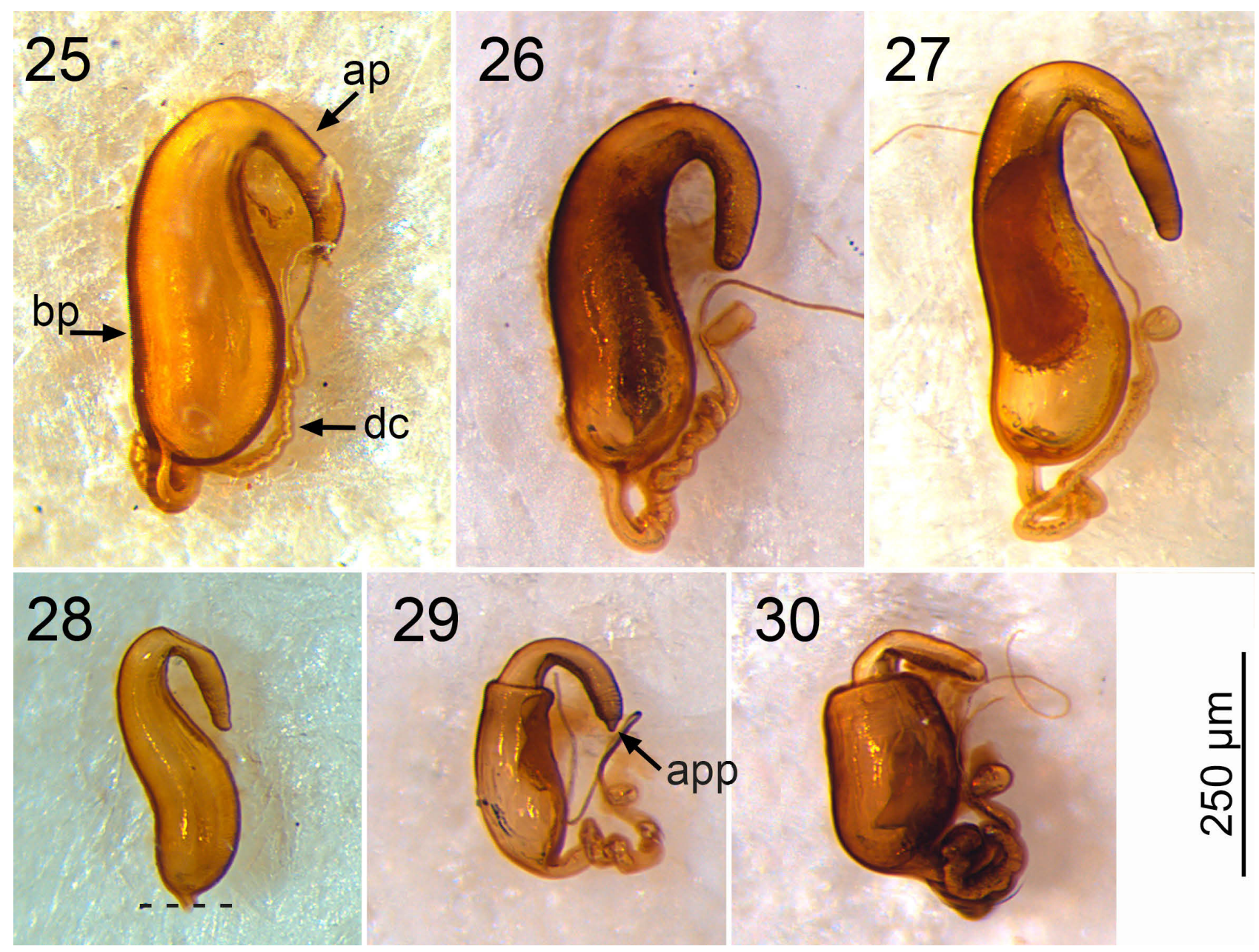

Figs 25-30. Spermatheca. 25 - Angulaphthona pelengensis (holotype of A. exalta), Democratic Republic of Congo, Kabwe; 26 - A. heteromorpha, "allotype"; 27 - A. latipennis, Yemen, Wadi Zabid; $28-A$. confusa sp. nov, paratype; $29-A$. schereri, Yemen, Wadi Zabid; 30 - A. violaceomicans, Sri Lanka. ap - apical part; app - appendix of distal part; bp - basal part; dc - ductus.

Remarks. The holotype (male) of $A$. pelengensis and the holotype (female) of $A$. exalta are considered conspecific due to the clear morphological similarity, specifically of the pronotum (Figs 11-12), strongly diagnostic for species of Angulaphthona. The precedence of the name Angulaphthona pelengensis Bechyné, 1960 is hereby established on the basis of the "Recommendation 24A. Action of First Reviser" of The International Code of Zoological Nomenclature (International Commission on Zoological Nomenclature, 1999). In fact, because $A$. exalta is known based only on one female and $A$. pelengensis on three males, and because the aedeagus is more diagnostic than the spermatheca in the genus Angulaphthona, it is our opinion that the name pelengensis "will best serve stability and universality of nomenclature" (International Commission on Zoological Nomenclature, 1999). Concerning the paratypes of $A$. exalta, the female from Kiamakoto-Kiwakishi was attributed to A. latipennis, while the female from Kaziba was not considered in our revision because it is immature.

A. pelengensis is easily distinguishable by the regular convexity of the pronotum and the pronotal punctation, clearly not homogenous, anteriorly smaller than posteriorly (Figs 11, 12). Also A. rossii sp. n. shows a similar pro- notal punctation (Fig. 13), but in this species a clear antebasal transversal depression on pronotum is clearly visible in lateral view, but absent in $A$. pelengensis. Median lobe of aedeagus (Fig. 21) in ventral view slightly narrower in the middle, with sub-rounded apex, and smooth surface; in lateral view, thick and slightly curved in basal half, and gradually thinner and sinuate in apical half; dorsal ligula with U-shaped base at apical fourth of aedeagus. Spermatheca (Fig. 25) thickest in basal part; basal and distal part not definitely separated; ductus inserted sub-apically, clearly coiled in proximal part.

Biometry. Male ( $\mathrm{n}=3$; range): $2.50 \leq \mathrm{LE} \leq 2.69 \mathrm{~mm} ; 1.80 \leq$ $\mathrm{WE} \leq 1.91 \mathrm{~mm} ; 0.75 \leq \mathrm{LP} \leq 0.78 \mathrm{~mm} ; 1.14 \leq \mathrm{WP} \leq 1.19 \mathrm{~mm}$; $2.05 \leq \mathrm{LAN} \leq 2.31 \mathrm{~mm} ; 1.23 \leq \mathrm{LAED} \leq 1.44 \mathrm{~mm} ; 3.22 \leq \mathrm{LB} \leq$ $3.72 \mathrm{~mm} ; 3.27 \leq \mathrm{LE} / \mathrm{LP} \leq 3.58 ; 1.55 \leq \mathrm{WE} / \mathrm{WP} \leq 1.62 ; 1.51 \leq$ $\mathrm{WP} / \mathrm{LP} \leq 1.52 ; 0.69 \leq \mathrm{WE} / \mathrm{LE} \leq 0.72 ; 0.62 \leq \mathrm{LAN} / \mathrm{LB} \leq 0.69$; $1.87 \leq \mathrm{LE} / \mathrm{LAED} \leq 2.05$.

Female $(\mathrm{n}=1): \mathrm{LE}=3.09 \mathrm{~mm} ; \mathrm{WE}=2.22 \mathrm{~mm} ; \mathrm{LP}=0.84 \mathrm{~mm}$; $\mathrm{WP}=1.31 \mathrm{~mm} ; \mathrm{LAN}=2.50 \mathrm{~mm} ; \mathrm{LSPC}=0.41 \mathrm{~mm} ; \mathrm{LB}=3.84$ $\mathrm{mm} ; \mathrm{LE} / \mathrm{LP}=3.67 ; \mathrm{WE} / \mathrm{WP}=1.69 ; \mathrm{WP} / \mathrm{LP}=1.56 ; \mathrm{WE} / \mathrm{LE}=$ $0.72 ; \mathrm{LAN} / \mathrm{LB}=0.65 ; \mathrm{LE} / \mathrm{LSPC}=7.62$.

Distribution. Democratic Republic of Congo, Uganda (Fig. 31). Central Afrotropical (CAT) chorotype. 
Ecological notes. Not available. The collecting sites are in Tropical Lowland Humid Forest and Tropical Lowland Grassland, Savanna \& Shrubland vegetation (Sayre et al., 2013).

\section{Angulaphthona rossii sp. $\mathbf{n}$.}

(Figs 5, 13, 22, 31)

ZooBank taxon LSID:

85FE84DC-2789-4DFD-A6B8-DD731BC60980

Diagnosis. Angulaphthona rossii sp. n. shares with $A$. pelengensis the uneven pronotal punctation, with punctures posteriorly wider than anteriorly (Figs 11-13). However, it is externally distinguishable by the transverse depression near the pronotal base, clearly visible in lateral view. The median lobe of aedeagus (Fig. 22) is clearly diagnostic as it has a strongly lanceolate shape, with widely rounded apex, in ventral view. Female unknown.

Description of the holotype $\hat{\sigma}$. Body sub-elliptical, moderately convex (Fig. 5); $\mathrm{LB}=3.61 \mathrm{~mm}$; maximum pronotal width in middle ( $\mathrm{WP}=1.25 \mathrm{~mm}$ ); maximum elytral width in middle $(\mathrm{WE}=2.00 \mathrm{~mm})$. Dorsum blackish, with blue metallic reflection, elytral disc slightly paler. Head with smooth surface, very finely wrinkled and micropunctate (Fig. 13); frontal carina distinctly raised, rounded apically, continuous with post-clypeal area; frontal tubercles V-shaped, moderately elongate, slightly raised; frontal grooves deeply impressed, from frontal tubercles to upper ocular margin. Antennae distinctly longer than $1 / 2$ body length $(\mathrm{LAN}=2.53 \mathrm{~mm} ; \mathrm{LAN} / \mathrm{LB}=0.70)$, dark brown, with paler insertion and first segment; LA: 100:63:79:100: 104:92:108:104:100:96:108. Pronotum (Fig. 13) slightly trapezoidal, distinctly transverse $(\mathrm{LP}=0.83 \mathrm{~mm}$; WP/LP $=$ 1.51 ), weakly rounded laterally; lateral margins moderately expanded; anterior angles slightly prominent; posterior angles with flat margins and distinctly prominent setigerous pore; antebasal part slightly depressed; pronotal surface weakly micropunctate, almost smooth, with moderately dense punctation; punctures small on most surfaces, bigger on antebasal part. Metathoracic wings macropterous. Elytra moderately elongate $(\mathrm{LE}=2.81 \mathrm{~mm} ; \mathrm{WE} / \mathrm{LE}=0.71)$, laterally slightly rounded (Fig. 5), with margin slightly expanded, visible in dorsal view (Fig. 13); punctation confused, similar to that on antebasal part of pronotum. Legs brownish; first pro- and mesotarsomeres distinctly dilated. Venter blackish; last visible abdominal ventrite with central lobe with a distinct median carina. Aedeagus (LAED $=1.22 \mathrm{~mm}$; LE/LAED = 2.31) (Fig. 22) in ventral view more enlarged apically than basally, slightly concave laterally; apical part sub-triangular, with rounded apex; ventral surface with two longitudinal depressions on apical third; dorsal surface with a median furrow on apical half; sulcus of dorsal ligula very short; in lateral view, aedeagus distinctly curved in basal $2 / 3 \mathrm{~s}$, than straight, with apical part slightly bent ventrally.

Type material. Holotype $\delta$ : Sierra Leone, M. ti Loma, Cascate Denkale [Loma Mts, $\left.9^{\circ} 10^{\prime} 02.03^{\prime \prime} \mathrm{N}, 11^{\circ} 07^{\prime} 14.43^{\prime \prime} \mathrm{W}\right], 750 \mathrm{~m}$, 2.i.1983, W. Rossi leg. (BAQ).

Type locality. Loma Mts (Sierra Leone).
Etymology. The specific epithet is after our colleague and friend Walter Rossi (University of L'Aquila), esteemed specialist in Laboulbeniales, who collected the only known specimen of this new species.

Distribution. Sierra Leone (Fig. 31). Northern-Western Afrotropical (NWA) chorotype.

Ecological notes. Not available. The site at which it was collected is in the area of Guineo-Congolian Evergreen \& Semi-Evergreen Rainforest vegetation (belonging to the Tropical Lowland Humid Forest) (Sayre et al., 2013).

\section{Angulaphthona schereri Gruev, 1981}

(Figs 6, 14, 17, 23, 29, 31)

Angulaphthona schereri Gruev, 1981: 56, 58.

Type material examined. Holotype $\delta$ : Yemen, Wadi Zabid

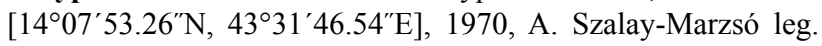
(HNHM). Paratypes: 6 and 2 , Yemen, same data as the holotype (HNHM).

Type locality. Wadi Zabid (Yemen).

Additional material examined. Ethiopia: Giuba [river] Margherita [lake] [6 $\left.24^{\prime} 46.76^{\prime \prime} \mathrm{N}, 37^{\circ} 43^{\prime} 16.17^{\prime \prime} \mathrm{E}\right]$, iv.1920, S. Patrizi leg., 1 spec. (MSNG); Madagascar: strada Ranohira-Ihosy (20

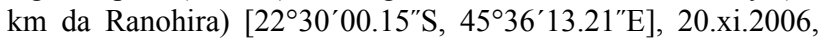
C. Canepari leg., 2 spec. (MSNG); $2.5 \mathrm{~km} \mathrm{NE}$ von Anara, SW von Iarintsena, ca $975 \mathrm{~m} \mathrm{NN}$, Buschtal, 21 $1^{\circ} 51^{\prime} 03^{\prime \prime} \mathrm{S}, 46^{\circ} 50^{\prime} 34^{\prime \prime} \mathrm{E}$, 6.xi.2003, U. Göllner leg., 1 spec. (ZMHB); Somalia: P.na di Fungalango $\left[\approx 0^{\circ} 04^{\prime} 04.94^{\prime \prime} \mathrm{N}, 42^{\circ} 35^{\prime} 31.90^{\prime \prime} \mathrm{E}\right]$, iii.-iv.1923, S. Patri-

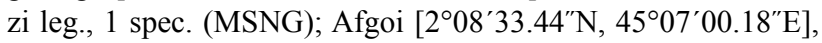
Agraria, iii.1986, C. Siegel leg., 1 spec. (MSNG); Brit. Somaliland $\left[\approx 10^{\circ} 14^{\prime} \mathrm{N}, 47^{\circ} 23^{\prime} \mathrm{E}\right]$, Hartan Pen., sweeping, 22.iii.1903, Dr. M. Cameron leg., 2 spec. (BMNH); ditto, Dolphin Bay $\left[\approx 10^{\circ} 14^{\prime} \mathrm{N}, 47^{\circ} 23^{\prime} \mathrm{E}\right], 1$ spec. (BMNH); Sudan: Upper Nile, Malakal, [9 $\left.30^{\prime} 28.40^{\prime \prime} \mathrm{N}, \quad 31^{\circ} 39^{\prime} 27.20^{\prime \prime} \mathrm{E}\right]$, 5.-20.i.1963, R. Linnavuori leg., 1 spec. (MZF); Singa Garden, on Erkovit hedge $\left[12^{\circ} 45^{\prime} 55.08^{\prime \prime} \mathrm{S}, 33^{\circ} 37^{\prime} 03.28^{\prime \prime} \mathrm{E}\right]$, 28.ii.1926, W. Ruttledge leg., 1 spec. (BMNH); Gendettu [Gandeto, $16^{\circ} 37^{\prime} 14^{\prime \prime} \mathrm{N}$, $\left.33^{\circ} 14^{\prime} 33^{\prime \prime} \mathrm{E}\right]$, in cotton fields, 20.x.1923, W.E. Giffard leg., 1 spec. (BMNH); Barakat $\left[14^{\circ} 18^{\prime} 48.05^{\prime \prime} \mathrm{N}, 33^{\circ} 31^{\prime} 39.58^{\prime \prime} \mathrm{E}\right]$, on nambook, 25.ii.1923, H.-B. Johnston leg., 1 spec. (BMNH); G.R.F. Medani [14 $\left.23^{\prime} 35.10^{\prime \prime} \mathrm{N}, 33^{\circ} 32^{\prime} 21.15^{\prime \prime} \mathrm{E}\right]$, feeding on Aristolochia bracteata, 6.iii.1922, H.W. Bedford leg., 1 spec.

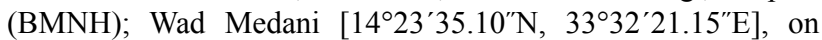
Aristolochia bracteata, 6.iii.1922, H.W. Bedford leg., 1 spec. (BMNH); ditto, on cotton, 6.xii.1922, 1 spec. (BMNH); Medani

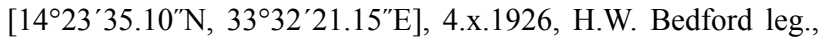
2 spec. (BMNH); Kadugli [ $\left.11^{\circ} 00^{\prime} 37.96^{\prime \prime} \mathrm{N}, 29^{\circ} 42^{\prime} 49.12^{\prime \prime} \mathrm{E}\right]$, on pea plant, 13.ii.1931, F.G.S. Whitfield leg., 1 spec. (BMNH); Yemen: Wadi Jaira, tributary of Wadi Siham, ca.3,000 ft [14 $\left.21^{\prime} 57^{\prime \prime} \mathrm{N}, 43^{\circ} 33^{\prime} 40^{\prime \prime} \mathrm{E}\right], 10.1 i i .1938$, H. Scott \& E.B. Britton leg., 5 spec. (BMNH); near Ta'izz, feald on road to Mocha, ca. $4,100 \mathrm{ft}\left[13^{\circ} 34^{\prime} 04.39^{\prime \prime} \mathrm{N}, 4^{\circ} 54^{\prime} 38.15^{\prime \prime} \mathrm{E}\right], 16 . x i i .1937, \mathrm{H}$. Scott \& E.B. Britton leg., 12 spec. (BMNH); Usaifira, 1 mile $\mathrm{N}$ of $\mathrm{Ta}^{\prime} \mathrm{izz}$, ca. $4,500 \mathrm{ft}\left[13^{\circ} 37^{\prime} 05.83^{\prime \prime} \mathrm{N}, 44^{\circ} 02^{\prime} 08.46^{\prime \prime} \mathrm{E}\right]$, in cultivated fields, 13.xii.1937, H. Scott \& E.B. Britton leg., 18 spec. (BMNH); ditto, 22.xii.1937, 3 spec. (BMNH); Hudaydah Bajil, [144ㄱ' $\left.27.28^{\prime \prime} \mathrm{N}, 42^{\circ} 58^{\prime} 15.18^{\prime \prime} \mathrm{E}\right]$, 18.v.1992, R. Linnavuori leg., 3 spec. (MZF); Wadi Surdud nr. Khamis Bani Said, [15 $\left.11^{\prime} 14.24^{\prime \prime} \mathrm{N}, 43^{\circ} 30^{\prime} 50.69^{\prime \prime} \mathrm{E}\right]$, 18.v.1992, R. Linnavuori leg.,

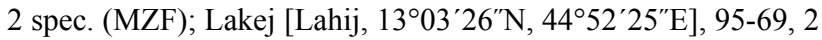
spec. (BMNH); Zambia: NE, $50 \mathrm{~km}$ W Kasama, [10³3'56.41"S, 30³9'00.89"E ], 26.xi.2004, M. Snížek \& V. Tichý leg., 2 spec. (BAQ); Chilinga Native Reserve [143' $43^{\prime \prime} \mathrm{S}, 30^{\circ} 22^{\prime} 30^{\prime \prime} \mathrm{E}$ ], on Citrus tree, 1 spec. (BMNH). 


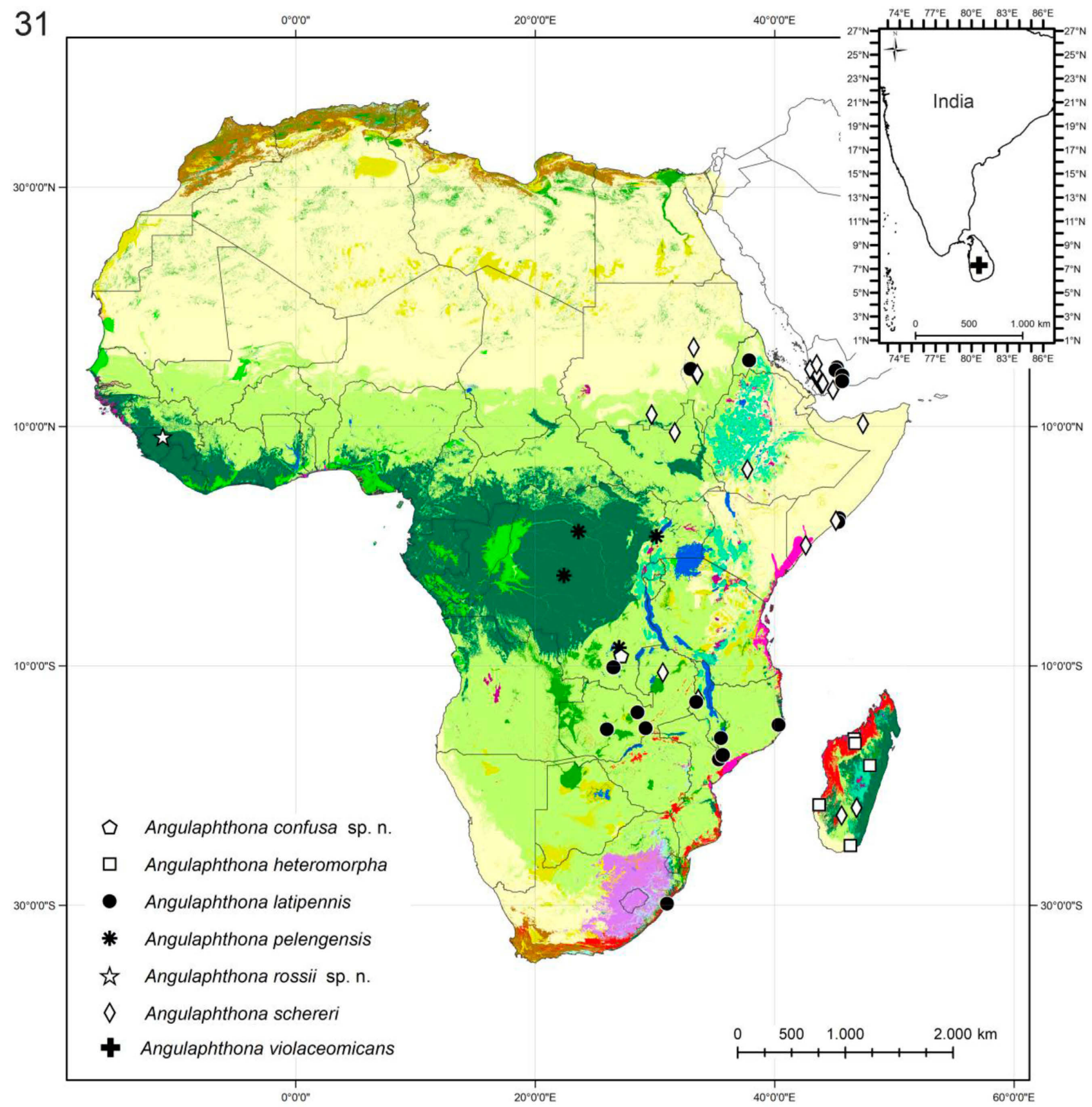

\section{Formations}

\begin{tabular}{|c|c|}
\hline Temperate Flooded \& Swamp Forest & Tropical Freshwater Marsh, Wet Meadow \& Shrubland \\
\hline Warm Temperate Forest & Tropical Montane Grassland \& Shrubland \\
\hline Temperate \& Boreal Cliff, Scree \& Other Rock Vegetation & Tropical Lowland Grassland, Savanna \& Shrubland \\
\hline Tropical Cliff, Scree \& Other Rock Vegetation & Mangrove \\
\hline Warm Desert \& Semi-Desert Scrub \& Grassland & Tropical Flooded \& Swamp Forest \\
\hline Salt Marsh & Tropical Montane Humid Forest \\
\hline Temperate \& Boreal Freshwater Marsh, Wet Meadow \& Shrubland & Tropical Lowland Humid Forest \\
\hline Temperate Grassland, Meadow \& Shrubland & Tropical Seasonally Dry Forest \\
\hline Mediterranean Scrub \& Grassland & Water \\
\hline
\end{tabular}

Fig. 31. Geographical distribution of the species of Angulaphtona relative to the types of African vegetation (Sayre et al., 2013). 
Remarks. A. schereri is recognizable by its pronotal punctation (Fig. 14), which is uniform and clearly impressed, about as large as that on elytra, and by the second, third and distal part of first antennomere distinctly paler than other antennal segments. Median lobe of aedeagus (Fig. 23) in ventral view fusiform, with sub-rounded apex and smooth surface; in lateral view slightly curved in basal half, then straight; dorsal ligula with U-shaped base at about apical fourth of aedeagus. Spermatheca (Fig. 29) with elongate, sub-cylindrical basal part, distinct from apical part; apical part curved, with a short appendix; ductus inserted apically, thick and coiled, except for the proximal part.

Biometry. Male ( $\mathrm{n}=10$; mean and standard deviation; range): $\mathrm{LE}=2.21 \pm 0.22 \mathrm{~mm}(1.91 \leq \mathrm{LE} \leq 2.53 \mathrm{~mm}) ; \mathrm{WE}=1.59 \pm 0.14$ $\mathrm{mm}(1.31 \leq \mathrm{WE} \leq 1.70 \mathrm{~mm}) ; \mathrm{LP}=0.70 \pm 0.05 \mathrm{~mm}(0.63 \leq \mathrm{LP} \leq$ $0.77 \mathrm{~mm}) ; \mathrm{WP}=1.02 \pm 0.10 \mathrm{~mm}(0.84 \leq \mathrm{WP} \leq 1.16 \mathrm{~mm}) ; \mathrm{LAN}$ $=1.84 \pm 0.17 \mathrm{~mm}(1.56 \leq \mathrm{LAN} \leq 2.03 \mathrm{~mm}) ; \mathrm{LAED}=1.07 \pm 0.15$ $\mathrm{mm}(0.81 \leq \mathrm{LAED} \leq 1.22 \mathrm{~mm}) ; \mathrm{LB}=2.99 \pm 0.23 \mathrm{~mm}(2.63 \leq \mathrm{LB}$ $\leq 3.28 \mathrm{~mm}) ; \mathrm{LE} / \mathrm{LP}=3.15 \pm 0.10(3.00 \leq \mathrm{LE} / \mathrm{LP} \leq 3.31) ; \mathrm{WE} / \mathrm{WP}$ $=1.55 \pm 0.07(1.45 \leq \mathrm{WE} / \mathrm{WP} \leq 1.70) ; \mathrm{WP} / \mathrm{LP}=1.46 \pm 0.05(1.35$ $\leq \mathrm{WP} / \mathrm{LP} \leq 1.51) ; \mathrm{WE} / \mathrm{LE}=0.72 \pm 0.03(0.67 \leq \mathrm{WE} / \mathrm{LE} \leq 0.77)$; $\mathrm{LAN} / \mathrm{LB}=0.61 \pm 0.03(0.56 \leq \mathrm{LAN} / \mathrm{LB} \leq 0.68) ; \mathrm{LE} / \mathrm{LAED}=$ $2.07 \pm 0.13(1.97 \leq \mathrm{LE} / \mathrm{LAED} \leq 2.31)$. Female $(\mathrm{n}=10$; mean and standard deviation; range): $\mathrm{LE}=2.41 \pm 0.21 \mathrm{~mm}(1.91 \leq \mathrm{LE}$ $\leq 2.66 \mathrm{~mm}) ; \mathrm{WE}=1.73 \pm 0.14 \mathrm{~mm}(1.38 \leq \mathrm{WE} \leq 1.91 \mathrm{~mm}) ; \mathrm{LP}$ $=0.73 \pm 0.06 \mathrm{~mm}(0.59 \leq \mathrm{LP} \leq 0.78 \mathrm{~mm}) ; \mathrm{WP}=1.10 \pm 0.09 \mathrm{~mm}$ $(0.88 \leq \mathrm{WP} \leq 1.19 \mathrm{~mm}) ; \mathrm{LAN}=1.89 \pm 0.14 \mathrm{~mm}(1.53 \leq \mathrm{LAN} \leq$ $2.02 \mathrm{~mm}) ; \mathrm{LSPC}=0.27 \pm 0.03 \mathrm{~mm}(0.23 \leq \mathrm{LSPC} \leq 0.31 \mathrm{~mm})$; $\mathrm{LB}=3.08 \pm 0.28 \mathrm{~mm}(2.50 \leq \mathrm{LB} \leq 3.56 \mathrm{~mm}) ; \mathrm{LE} / \mathrm{LP}=3.32 \pm$ 0.09 (3.21 $\leq \mathrm{LE} / \mathrm{LP} \leq 3.43) ; \mathrm{WE} / \mathrm{WP}=1.57 \pm 0.02(1.55 \leq \mathrm{WE} /$ $\mathrm{WP} \leq 1.61) ; \mathrm{WP} / \mathrm{LP}=1.51 \pm 0.03(1.47 \leq \mathrm{WP} / \mathrm{LP} \leq 1.57) ; \mathrm{WE} /$ $\mathrm{LE}=0.72 \pm 0.01(0.70 \leq \mathrm{WE} / \mathrm{LE} \leq 0.74) ; \mathrm{LAN} / \mathrm{LB}=0.61 \pm 0.03$ $(0.55 \leq \mathrm{LAN} / \mathrm{LB} \leq 0.65) ; \mathrm{LE} / \mathrm{LSPC}=8.90 \pm 1.13(6.42 \leq \mathrm{LE} /$ LSPC $\leq 10.67)$.

Distribution. Ethiopia, Madagascar, Somalia, Sudan, Yemen, Zambia. Eastern Afrotropical (EAF) chorotype, with expansion to Madagascar.

Ecological notes. Collected in cultivated fields, on Citrus (Rutaceae), Aristolochia bracteata (Aristolochiaceae), cotton (Malvaceae), pea plants (Fabaceae). The collecting sites are located in very different types of vegetation belonging to the Tropical Lowland Grassland, Savanna \& Shrubland and the Warm Desert \& Semi-Desert Scrub \& Grassland formations (Sayre et al., 2013).

\section{Angulaphthona violaceomicans (Chen, 1936) comb. $\mathbf{n}$.}

(Figs 7, 15, 24, 30, 31)

Aphthona violaceomicans Chen, 1936: 83; Kimoto, 2003: 37.

Type material examined. Holotype $\delta$ : [Sri Lanka] Kandy [7¹7'54.12"N, 80³8'38.78"E], W. Horn, 1899 (SDEI).

Type locality. Kandy (Sri Lanka).

Additional material examined. Sri Lanka: "Thwaites 67-25" (BMNH) [Accession registry 1867-25 of BMNH reports: "Ceylon, presented by Dr. G.H.K. Thwaites"], 7 spec.

Remarks. We here transfer the species Aphthona violaceomicans to the genus Angulaphthona, based on the diagnostic characters of this genus (see above).
A. violaceomicans is easily recognizable by the very expanded lateral margin of elytra and the clearly transverse pronotum (Fig. 15). It is also smaller in size than the other species $(\mathrm{LE}+\mathrm{LP} \leq 2.47 \mathrm{~mm}$ in male, $=2.39 \mathrm{~mm}$ in female). Median lobe of aedeagus (Fig. 24) in ventral view laterally sub-parallel, with smooth surface and a thin, short median sulcus on apical part; apex sub-triangular, with small, pointed, lateral expansions; in lateral view clearly curved in basal $2 / 3 \mathrm{~s}$, then straight; dorsal surface slightly depressed medially; dorsal ligula U-shaped, with base at apical fourth of aedeagus. Spermatheca (Fig. 30) with a very thick, sub-cylindrical basal part, distinct from apical part; distal part with a short appendix; ductus inserted sublaterally, very thick and with many coils.

Biometry. Male ( $\mathrm{n}=6$; mean and standard deviation; range): $\mathrm{LE}=1.76 \pm 0.09 \mathrm{~mm}(1.66 \leq \mathrm{LE} \leq 1.91 \mathrm{~mm}) ; \mathrm{WE}=1.30 \pm 0.04$ $\mathrm{mm}(1.25 \leq \mathrm{WE} \leq 1.34 \mathrm{~mm}) ; \mathrm{LP}=0.52 \pm 0.02 \mathrm{~mm}(0.50 \leq \mathrm{LP} \leq$ $0.56 \mathrm{~mm}) ; \mathrm{WP}=0.86 \pm 0.04 \mathrm{~mm}(0.81 \leq \mathrm{WP} \leq 0.94 \mathrm{~mm}) ; \mathrm{LAN}$ $=1.57 \pm 0.12 \mathrm{~mm}(1.38 \leq \mathrm{LAN} \leq 1.72 \mathrm{~mm}) ; \mathrm{LAED}=0.87 \pm$ $0.02 \mathrm{~mm}(0.84 \leq \mathrm{LAED} \leq 0.91 \mathrm{~mm}) ; \mathrm{LB}=2.23 \pm 0.12 \mathrm{~mm}(2.11$ $\leq \mathrm{LB} \leq 2.45 \mathrm{~mm}) ; \mathrm{LE} / \mathrm{LP}=3.37 \pm 0.09$ (3.21 $\leq \mathrm{LE} / \mathrm{LP} \leq 3.47)$; $\mathrm{WE} / \mathrm{WP}=1.52 \pm 0.04(1.43 \leq \mathrm{WE} / \mathrm{WP} \leq 1.56) ; \mathrm{WP} / \mathrm{LP}=1.64 \pm$ $0.04(1.59 \leq \mathrm{WP} / \mathrm{LP} \leq 1.69) ; \mathrm{WE} / \mathrm{LE}=0.74 \pm 0.02(0.70 \leq \mathrm{WE} /$ $\mathrm{LE} \leq 0.75) ; \mathrm{LAN} / \mathrm{LB}=0.70 \pm 0.03(0.65 \leq \mathrm{LAN} / \mathrm{LB} \leq 0.73) ; \mathrm{LE} /$ $\mathrm{LAED}=2.03 \pm 0.07(1.93 \leq \mathrm{LE} / \mathrm{LAED} \leq 2.11)$. Female $(\mathrm{n}=1)$ : $\mathrm{LE}=1.84 \mathrm{~mm} ; \mathrm{WE}=1.34 \mathrm{~mm} ; \mathrm{LP}=0.55 \mathrm{~mm} ; \mathrm{WP}=0.91 \mathrm{~mm}$; $\mathrm{LAN}=1.61 \mathrm{~mm} ; \mathrm{LSPC}=0.27 \mathrm{~mm} ; \mathrm{LB}=2.38 \mathrm{~mm} ; \mathrm{LE} / \mathrm{LP}=$ 3.37; WE/WP $=1.48 ; \mathrm{WP} / \mathrm{LP}=1.66 ; \mathrm{WE} / \mathrm{LE}=0.73 ; \mathrm{LAN} / \mathrm{LB}=$ $0.68 ; \mathrm{LE} / \mathrm{LSPC}=6.94$.

Distribution. Sri Lanka (Fig. 31). The peculiar distribution of this species is discussed further in the Conclusions.

Ecological notes. Not available.

\section{Key to species of Angulaphthona}

1 Smaller size: $\mathrm{LE}+\mathrm{LP}<2.50 \mathrm{~mm}$ in male, $<2.50 \mathrm{~mm}$ in female. Lateral margin of elytra very expanded (Fig. 15). Median lobe of aedeagus (Fig. 24) apically sub-triangular, with small, pointed, lateral expansions, and with a thin, short ventral sulcus; in lateral view clearly curved in basal half, then straight. Spermatheca (Fig. 30) with very thick basal part, distinct from apical part, and very thick, multicoiled ductus ..... A. violaceomicans

- Larger size: $\mathrm{LE}+\mathrm{LP} \geq 2.50 \mathrm{~mm}$ in male, $\geq 2.50 \mathrm{~mm}$ in female. Lateral margins of elytra from weakly to distinctly expanded (Figs 8-14).....

2 Pronotal punctation anteriorly clearly smaller than posteriorly (Figs 11-13) ............................................................... 3

- Pronotal punctation uniform in size and distribution (Figs 8-10, 14-15).....

3 Antebasal part of pronotum slightly depressed (clearly visible in lateral view). Median lobe of aedeagus (Fig. 22) in ventral view apically clearly wider than basally, and in lateral view evenly thick. Female unknown.......................... rossii sp. n. - Pronotum evenly convex. Median lobe of aedeagus (Fig. 21) in ventral view apically as wide as basally, and in lateral view clearly thinner in apical part. Spermatheca (Fig. 25) with thick basal part, and ductus inserted sub-apically, clearly coiled proximally ........................................... A. pelengensis

4 Pronotal punctation clearly impressed, about as large as that on elytra (Fig. 14). Second, third and distal part of first antennomere distinctly paler. Median lobe of aedeagus (Fig. 23) fusiform in ventral view; in lateral view slightly curved in 
basal half, then straight. Spermatheca (Fig. 29) with basal and apical part clearly distinct. A. schereri

- Pronotal punctation weakly impressed and smaller than on elytra (Figs 8-10). Antennal segments more uniform in colour. Spermatheca with basal and apical part not distinctly separated (Figs 26-28).

5 Pronotal and elytral punctation very weakly impressed (Fig. 9). Pronotum sub-rectangular (Fig. 9). Median lobe of aedeagus (Fig. 19) apically with two lateral, pointed expansions, and a large, rounded, flattened median tooth; in lateral view very thick and clearly curved. Spermatheca (Fig. 26) slightly variable, with ductus weakly coiled or clearly and unevenly coiled over most its length..... A. heteromorpha

- Pronotal and elytral punctation more distinctly impressed (Figs 8, 10). Pronotum sub-trapezoidal (Figs 8, 10)............. 6

6 Larger size: $\mathrm{LE}+\mathrm{LP} \geq 3.34 \mathrm{~mm}$ in male, $\geq 3.44 \mathrm{~mm}$ in female. Pronotum with sub-rounded anterior angles (Fig. 10). Median lobe of aedeagus (Fig. 20) in ventral view clearly and abruptly wider in apical $2 / 5 \mathrm{~s}$; in lateral view with apical part distinctly bent ventrally. Spermatheca (Fig. 27) with ductus loosely and irregularly coiled in proximal part

A. latipennis

- Smaller size: $\mathrm{LE}+\mathrm{LP}<3.34 \mathrm{~mm}$ in male, $<3.44 \mathrm{~mm}$ in $\mathrm{fe}-$ male. Pronotum with dentiform anterior angles (Fig. 8). Median lobe of aedeagus (Fig. 18) in ventral view basally and apically sub-parallel, but clearly enlarged near middle; in lateral view with apical part distinctly bent dorsally. Ductus of spermatheca damaged and its morphology unknown.

A. confusa sp. $\mathrm{n}$

\section{DISCUSSION}

Based on the present revision, the flea beetle genus $\mathrm{An}$ gulaphthona currently comprises 7 species, of which two are new to science. They do not appear to be common based on the rather low number of specimens (for most species) in the main African entomological collections in the World. Most species occur in Central and Eastern Africa with extensions to the near Arabian Peninsula; two in Madagascar, one of which is endemic; one in Western Africa. A further species, $A$. violaceomicans, is known from Sri Lanka. There are another three genera of Alticini occurring both in the Afrotropical and the Oriental regions, Chabria Jacoby, Jacobyana Maulik, and Sanckia Duvivier (Biondi \& D'Alessandro, 2011, 2012, 2013). Based on the present data, we cannot be sure whether Sri Lanka is part of the natural distribution of this genus or if this species was imported there from Africa on exotic plants. Different hypotheses can be put forward. If Sri Lanka is part of the natural distribution of the genus Angulaphthona then: (a) $A$. violaceomicans is probably a widespread (even though not common) species that has not been found yet elsewhere on the Indian sub-continent; (b) A. violaceomicans is the result of the differentiation of an ancestral widespread species reaching southern Asia through Madagascar. In fact, two Angulaphthona species occur in Madagascar: A. heteromorpha, endemic to the island, and the more widespread A. schereri. The similarities between the latter species and A. violaceomicans in spermatheca (they are the only two species with apical and basal part clearly distinct, and with a short appendix in the distal part) and in the last visible ventrite of males (they are the only two species without the typical carinate central lobe) might be important clues to their relationships. In this case, we do not expect to find A. violaceomicans in Africa and Madagascar. However, a different hypothesis could be that Sri Lanka is not part of the natural distribution of the genus, with $A$. violaceomicans imported to Kandy, the only known locality, on some exotic plants. The city of Kandy hosts the Udawatta Kele Sanctuary, a historic forest reserve on a hill-ridge that is heavily invaded by exotic trees and species of creeper (Nyanatusita \& Dissanayake, 2013). Kimoto (2003) reports eight specimens of $A$. violaceomicans (as Aphthona) specifically from Udawatta Kele Sanctuary. In addition, the fact that Dr. G.H.K. Thwaites, who presented the seven specimens preserved in $\mathrm{BMNH}$, was the director of this botanical reserve for long time, makes it plausible that the specimens were collected there. However, this species was not reported by Scherer (1969) among the flea beetles from the Indian sub-continent. This "Africa to Asia" import hypothesis would be confirmed if $A$. violaceomicans was found in Africa and/or Madagascar in the future.

ACKNOWLEDGEMENTS. We are grateful to the institutions that allowed us to study their material, and particularly to M. Balke and D.F. Amran Balke (ZSM), M. Geiser (BMNH), B. Grobbelaar (SANC), A. Mantilleri (MNHN), O. Merkl (HNHM), K. Nadein (SDEI), R. Poggi (MSNG), D. Van den Spiegel (RMCA). Thanks to our colleague J. Bezděk for his suggestions and data from his collection.

\section{REFERENCES}

Alfieri A. 1976: The Coleoptera of Egypt. - Mém. Soc. Entomol. Egypte 5: $\mathrm{x}+361 \mathrm{pp}$.

Bechyné J. 1955: Description de deux nouveaux Alticides de Madagascar (Col. Phytophaga). - Nat. Malgache 7: 61-62.

Bechyné J. 1959: Coleoptera: Chrysomelidae II. In Hanström B., Brinck P. \& Rudebeck G. (eds): South African Animal Life. Results of the Lund University Expedition in 1950-1951, Vol. 6. Almqvist \& Wiksell, Stockholm, pp. 227-238.

Bechyné J. 1960: Alticidae (Coleoptera, Phytophagoidea). In: Exploration du Parc National de l'Upemba. Mission G.F. de Witte en Collaboration avec W. Adam, A. Janssens, L. Van Meel et R. Verheyen (1946-1949). Fasc. 59 [1959]. Institut des Parcs Nationaux du Congo Belge, Bruxelles, pp. 39-114.

Biondi M. \& D'Alessandro P. 2006: Biogeographical analysis of the flea beetle genus Chaetocnema in the Afrotropical Region: distribution patterns and areas of endemism. $-J$. Biogeogr. 33: 720-730.

Biondi M. \& D'AlessandRo P. 2008: Taxonomical revision of the Longitarsus capensis species-group: An example of Mediterranean-southern African disjunct distributions (Coleoptera: Chrysomelidae). — Eur. J. Entomol. 105: 719-736.

Biondi M. \& D'Alessandro P. 2010a: Genus-group names of Afrotropical flea beetles (Coleoptera: Chrysomelidae: Alticinae): Annotated catalogue and biogeographical notes. - Eur. J. Entomol. 107: 401-424.

Biondi M. \& D'AlessAndRo P. 2010b: Revision of the Afrotropical flea beetle genus Serraphula Jacoby and description of Bechynella, a new genus from Western and Central Africa (Coleoptera: Chrysomelidae: Alticinae). — Zootaxa 2444: 1-44.

Biondi M. \& D'Alessandro P. 2011: Jacobyana Maulik, an Oriental flea beetle genus new for the Afrotropical Region with description of three new species from Central and Southern Af- 
rica (Coleoptera, Chrysomelidae, Alticinae). - ZooKeys 86 47-59.

Biondi M. \& D'AlessandRo P. 2012: Afrotropical flea beetle genera: a key to their identification, updated catalogue and biogeographical analysis (Coleoptera, Chrysomelidae, Galerucinae, Alticini). - Zookeys 253: 1-158.

Biondi M. \& D’Alessandro P. 2013a: The genus Chabria Jacoby: first records in the Afrotropical region with description of three new species and annotated worldwide species catalogue (Coleoptera, Chrysomelidae, Galerucinae, Alticini). — Zool. Anz. 252: 88-100.

Biondi M. \& D'Alessandro P. 2013b: Ntaolaltica and Pseudophygasia, two new flea beetle genera from Madagascar (Coleoptera: Chrysomelidae: Galerucinae: Alticini). — Insect Syst. Evol. 44: 93-106.

Biondi M. \& D'Alessandro P. 2015: Revision of the Afrotropical genus Notomela Jacoby, 1899 with description of $N$. joliveti sp. n. from Principe Island (Coleoptera, Chrysomelidae, Galerucinae, Alticini). In Jolivet P., Santiago-Blay J. \& Schmitt M (eds): Research on Chrysomelidae 5. - ZooKeys 547: 63-74.

Biondi M. \& D'Alessandro P. 2016: Revision of Diphaulacosoma Jacoby, an endemic flea beetle genus from Madagascar, with description of three new species (Coleoptera: Chrysomelidae, Galerucinae, Alticini). - Fragm. Entomol. 48: 143-151.

Biondi M. \& D'Alessandro P. 2017: Guilielmia Weise, a little known Afrotropical flea beetle genus: systematic affinities and description of a second new species from Central Africa (Coleoptera, Chrysomelidae, Galerucinae, Alticini). - Zootaxa 4323: 572-578.

Biondi M., Frasca R., Grobbelaar E. \& D’Alessandro P. 2017 Supraspecific taxonomy of the flea beetle genus Blepharida Chevrolat, 1836 (Coleoptera: Chrysomelidae) in the Afrotropical Region and description of Afroblepharida subgen. nov. Insect Syst. Evol. 48: 97-155.

Bouchard P., Bousquet Y., Davies A.E., Alonso-Zarazaga M.A., Lawrence J.F., Lyal C.H.C., Newton A.F., Reid C.A.M., SchmitT M., ŚLIPIŃSKI S.A. \& SMITH A.B.T. 2011: Family-group names in Coleoptera (Insecta). - ZooKeys 88: 1-972.

Bryant G.E. 1957: Chrysomelidae of South-West Arabia. Ann. Mag. Nat. Hist. (Ser. 12) 10: 353-363.

CHEN S.H. 1936: Notes on some flea-beetles from Tropical Asia (II). - Sinensia 7: 80-88.

Chevrolat L.A.A. 1836: Chrysomelidae. In Dejean P.F.M.A. (ed.): Catalogue des Coléoptères de la Collection de M. le Comte Dejean. 2nd ed. Part 5. Librairie Méquignon-Marvis Père et Fils, Paris, pp. 361-442.

D'Alessandro P., Urbani F. \& Biondi M. 2014: Biodiversity and biogeography in Madagascar: revision of the endemic flea beetle genus Neodera Duvivier, 1891 with description of 19 new species (Coleoptera, Chrysomelidae, Galerucinae, Alticini). Syst. Entomol. 39: 710-748.

D'Alessandro P., Samuelson A. \& Biondi M. 2016: Taxonomic revision of the genus Arsipoda Erichson, 1842 (Coleoptera, Chrysomelidae) in New Caledonia. - Eur. J. Taxon. 230: $1-61$.

D’Alessandro P., Frasca R., Grobbelaar E., Iannella M. \& BIONDI M. 2017: Systematics and biogeography of the Afrotropical flea beetle subgenus Blepharidina (Afroblepharida) Biondi \& D'Alessandro, with description of seven new species (Coleoptera, Chrysomelidae, Galerucinae, Alticini). - Insect Syst. Evol. (prepublished online, 38 pp.); doi: 10.1163/1876312X00002182

DÖBERL M. 1986: Die spermathek als bestimmungshilfe bei den Alticinen. - Entomol. Blätt. 82: 3-14.
DöBERL M. 2010: Beitrag zur Kenntnis der afrotropischen Arten von Altica Geoffroy, 1762 unter Ausschluss der Arten Madagaskars (Coleoptera: Chrysomelidae: Alticinae). - Dt. Entomol. Z. 120: 51-72.

EvenHuis N.L. 2016: The Insect and Spider Collections of the World Website. URL: http://hbs.bishopmuseum.org/codens/ (last accessed 8 Oct. 2017).

FURTH D.G. 1980: Inter-generic differences in the metafemoral apodeme of flea beetles (Chrysomelidae: Alticinae). - Syst. Entomol. 5: 263-271.

FurTH D.G. \& SUzUKI K. 1994: Character correlation studies of problematic genera of Alticinae in relation to Galerucinae (Coleoptera: Chrysomelidae). In Furth D.G. (ed.): Proceedings of the Third International Symposium on the Chrysomelidae, Beijing, 1992. Backhuys, Leiden, pp. 116-135.

FurTh D.G. \& SuzuKi K. 1998: Studies of Oriental and Australian Alticinae genera based on the comparative morphology of the metafemoral spring, genitalia, and hind wing venation. In Biondi M., Daccordi M. \& Furth D.G. (eds): Proceedings of the Fourth International Symposium on the Chrysomelidae. Proceedings of XX International Congress of Entomology, Firenze, 1996. Museo Regionale di Scienze Naturali, Torino, pp. 91-124.

GENTRY J.W. 1965: Crop Insects of Northeast Africa-Southwest Asia. Agriculture Handbook 273. Agricultural Research Service, USDA, Washington, D.C., ii +210 pp.

Gruev B. 1981: Bemerkungen über die Arten der Gattung Angulaphthona Bechyné, 1960, mit Beschreibung einer neuen Art aus Jemen (JAR) (Coleoptera, Chrysomelidae, Alticinae). Reichenbachia 19(10): 55-58.

HeiKertinger F. 1944: Bestimmungstabelle der paläarktischen Aphthona-Arten. - Koleopt. Rundsch. 30: 37-122.

HeIKertinger F. \& Csiki C. 1940: Coleopterorum Catalogus Auspiciis et Auxilio W. Junk Editus a S. Schenkling. Partes 160 et 169. Chrysomelidae: Halticinae, Vol. 25. Dr. W. Junk, s-Gravenhage, pp. 337-635.

InTERNATIONAL COMMISSION ON ZoOlogical Nomenclature 1999: The International Code of Zoological Nomenclature. 4th ed. The Natural History Museum, London, xxix $+306 \mathrm{pp}$.

Кімото S. 2003: The Chrysomelidae (Insecta: Coleoptera) collected by Dr. Akio Otake, on the occasion of his entomological survey in Sri Lanka from 1973 to 1975. - Bull. Kitakyushu Mus. Nat. Hist. Hum. Hist. (A) 1: 23-43.

Konstantinov A.S. \& Vandenberg N.J. 1996: Handbook of Palearctic flea beetle (Coleoptera: Chrysomelidae: Alticinae). Contrib. Entomol. 1: 237-439.

Medvedev L.N. 1996: The Chrysomelidae of Arabia. - Fauna of Saudi Arabia 15: 215-263.

NADEIN K.S. 2012: Catalogue of Alticini genera of the World (Coleoptera: Chrysomelidae). Beetles and Coleopterists website, Zoological Institute, Saint-Petersburg. URL: http://www.zin. ru/Animalia/Coleoptera/eng/alticinw.htm (last accessed 8 Oct. 2017).

NADEIN K. \& Betz O. 2016: Jumping mechanisms and performance in beetles. I. Flea beetles (Coleoptera: Chrysomelidae: Alticini). - J. Exp. Biol. 219: 2015-2027.

NADEIN K.S. \& BEZDĚK J. 2014: 2.7.8. Galerucinae Latreille 1802. In Leschen R.A.B. \& Beutel R.G. (eds): Handbook of Zoology, Vol. 4/40: Coleoptera, Beetles, Vol. 3: Morphology and Systematics (Phytophaga). Walter de Gruyter, Berlin, pp. 251-259.

Nyanatusita B. \& Dissanayake R. 2013: Udawattakele: A sanctuary destroyed from within. - Loris J. Wildlife Nat. Prot. Soc. Sri Lanka 26(5-6): 38-47.

PIC M. 1921: Aphthona latipennis n. sp. — L'Echange 37(406): 15. 
Pollard D.G. 1957: Halticinae of the Sudan. - Bull. Entomol. Res. 47: 73-87.

Sayre R., Comer P., Hak J., Josse C., Bow J., Warner H., Larwanou M. , Kelbessa E., Bekele T., Kehl H. et al. 2013: $A$ New Map of Standardized Terrestrial Ecosystems of Africa. Association of American Geographers, Washington, DC, 24 pp.

SCHERER G. 1959: Die Alticiden-Ausbeute der Expedition des Museums G. Frey nach Nigeria-Kamerun 1955/56 (Col. Phytoph.). - Entomol. Arb. Mus. G. Frey 10: 177-265.

SCHERER G. 1961: Liste der von Herrn P. Renaud im Tchad-Gebiet gesammelten Alticiden (Coleoptera, Chrysomelidae, Alticinae). - Rev. Zool. Bot. Afr. 64: 172-174.

Scherer G. 1962a: Alticinae (Coleoptera, Phytophaga) Fam. Chrysomelidae. In: Exploration du Parc National de la Garamba. Mission H. De Saeger en Collaboration avec P. Baert, G. Demoulin, I. Denisoff, J. Martin, M. Micha, A. Noirfalise, P. Schoemaker, G. Troupin et J. Verschuren (1949-1952). Fasc. 31. Institut des Parcs Nationaux du Congo et du Rwanda, Bruxelles, pp. 3-86.
SCHERER G. 1962b: Beitrag zur Kenntnis der Alticiden-fauna Zentral-Afrikas (Coleoptera, Chrysomelidae, Alticinae). - Ann. Mus. R. Afr. Centr. (Tervuren) (Ser. 8, Sci. Zool.) 113: 7-82.

SCHERER G. 1969: Die Alticinae Des Indischen Subkontinentes (Coleoptera: Chrysomelidae). - Pacif. Insects Monogr. 22: $1-251$.

SCHERER G. 1978: Missione 1965 del professor Giuseppe Scortecci nello Yemen (Arabia meridionale). Coleoptera: Chrysomelidae: Alticinae. - Atti Soc. Ital. Sci. Nat. Mus. Civ. Storia Nat. Milano 119: 264-266.

SUZUKI K. 1988: Comparative morphology of the internal reproductive system of the Chrysomelidae (Coleoptera). In Jolivet P., Petitpierre E. \& Hsiao T.H. (eds): Biology of Chrysomelidae. Series Entomologica 42. Kluwer Academic, Dordrecht, pp. 317-355.

Received November 6, 2017; revised and accepted December 20, 2017 Published online February 9, 2018 